\title{
ELLIPTIC CURVES OVER REAL QUADRATIC FIELDS ARE MODULAR
}

\author{
NUNO FREITAS, BAO V. LE HUNG, SAMIR SIKSEK
}

Abstract. We prove that all elliptic curves defined over real quadratic fields are modular.

\section{Contents}

\section{Part 1. Introduction}

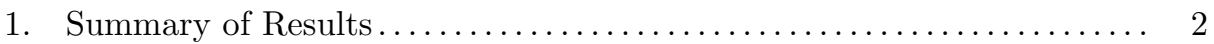

Part 2. Background 5

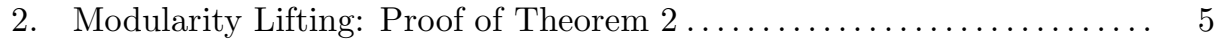

3. Background on Modular Curves ........................... 6

3.1. Borel Subgroups, Cartan Subgroups, and Normalizers............. 7

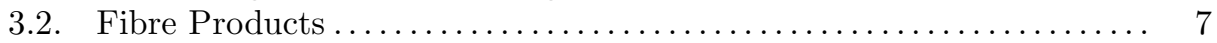

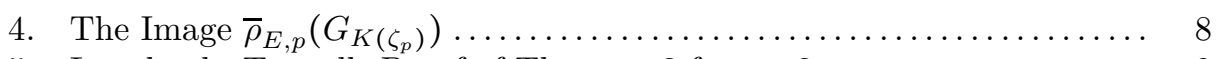

5. Langlands-Tunnell: Proof of Theorem 3 for $p=3 \ldots \ldots \ldots \ldots \ldots \ldots$

Part 3. Modularity Switching 9

6. 3-5 Modularity Switching: Proof of Theorem 3 for $p=5 \ldots \ldots \ldots \ldots .9$

7. 3-7 Modularity Switching: Proof of Theorem $4 \ldots \ldots \ldots \ldots \ldots \ldots \ldots$

8. On Modularity over Totally Real Fields: Proof of Theorem $5 \ldots \ldots \ldots .15$

Part 4. A More Precise Study of Images 15

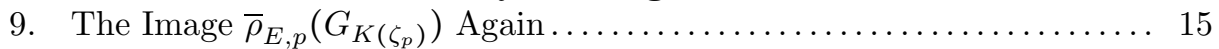

10. Modular Curves Again ................................... 16

11. Theorem 1 follows from Lemmas 1.1 and $1.2 \ldots \ldots \ldots \ldots \ldots \ldots \ldots$

Part 5. Real Quadratic Points on Certain Modular Curves 18

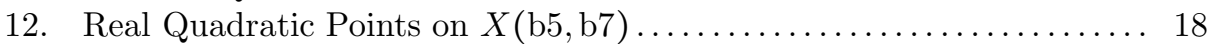

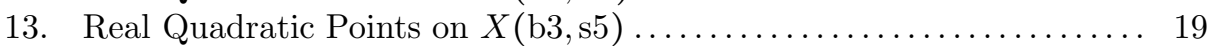

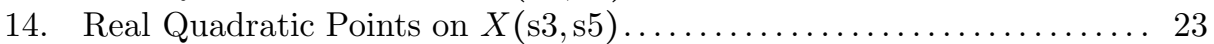

15. Models for some Modular Curves of Genus $1 \ldots \ldots \ldots \ldots \ldots \ldots \ldots . \ldots 25$

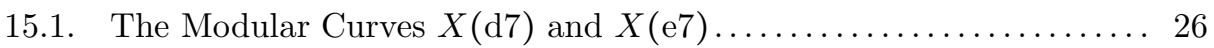

Date: July 21, 2014.

2010 Mathematics Subject Classification. Primary 11F80, Secondary 11G05.

Key words and phrases. modularity, elliptic curves, totally real fields.

The first-named author is supported through a grant within the framework of the DFG Priority Programme 1489 Algorithmic and Experimental Methods in Algebra, Geometry and Number Theory. The third-named author is supported by an EPSRC Leadership Fellowship EP/G007268/1, and EPSRC LMF: L-Functions and Modular Forms Programme Grant EP/K034383/1. 
15.2. The Modular Curves $X(\mathrm{~b} 3, \mathrm{~b} 5)$ and $X(\mathrm{~s} 3, \mathrm{~b} 5) \ldots \ldots \ldots \ldots \ldots \ldots \ldots$

16. A Sieve for the Symmetric Square of a Fibre Product............. 29

16.1. Points on the Normalization of a Fibre Product.............. 30

17. Real Quadratic Points on $X(\mathrm{~b} 3, \mathrm{~b} 5, \mathrm{~d} 7), X(\mathrm{~s} 3, \mathrm{~b} 5, \mathrm{~d} 7), X(\mathrm{~b} 3, \mathrm{~b} 5, \mathrm{e} 7)$,

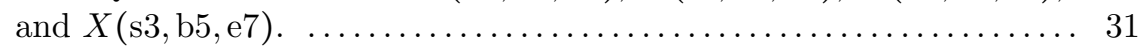

18. Modularity of Elliptic Curves over $\mathbb{Q}(\sqrt{5}) \ldots \ldots \ldots \ldots \ldots \ldots \ldots \ldots \ldots$

Part 6. Further Directions 35

19. On Modularity over Totally Real Fields (Again) ............... 35

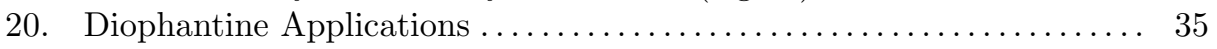

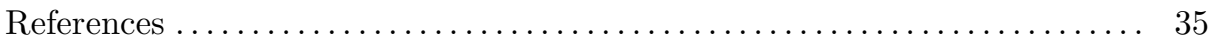

\section{Part 1. Introduction}

\section{Summary of Results}

One of the great achievements of modern number theory is the proof by Breuil, Conrad, Diamond and Taylor [9] of the Shimura-Taniyama (or modularity) conjecture for elliptic curves over $\mathbb{Q}$. This built on the earlier pioneering work of Wiles [83, and of Taylor and Wiles [81, which established the modularity of semistable elliptic curves over $\mathbb{Q}$, and was historically the final step in the proof of Fermat's Last Theorem. In this paper we prove the analogous modularity statement for elliptic curves over real quadratic fields.

Let $K$ be a totally real number field, and write $G_{K}:=\mathrm{Gal}(\overline{\mathbb{Q}} / K)$ for its absolute Galois group. Let $E$ be an elliptic curve over $K$. Recall that $E$ is modular if there exists a Hilbert cuspidal eigenform $\mathfrak{f}$ over $K$ of parallel weight 2, with rational Hecke eigenvalues, such that the Hasse-Weil L-function of $E$ is equal to the L-function of $\mathfrak{f}$. A more conceptual way to phrase this is that there is an isomorphism of compatible systems of Galois representations

$$
\rho_{E, p} \cong \rho_{\mathfrak{f}, p}
$$

where the left-hand side is the Galois representation arising from the action of $G_{K}$ on the $p$-adic Tate module $T_{p}(E)$, while the right-hand side is the Galois representation associated to $\mathfrak{f}$ by Eichler and Shimura (when $K=\mathbb{Q}$ ) [25], and by Carayol [12] 13, Blasius and Rogawski [8], Wiles [84] and Taylor [79] (for general totally real $K$ ).

Work of Jarvis and Manoharmayum [0] establishes modularity of semistable elliptic curves over $\mathbb{Q}(\sqrt{2})$ and $\mathbb{Q}(\sqrt{17})$, and there are various other works $[1],[26$, which establish modularity under local assumptions on the curve $E$ and the field $K$. In this paper, we prove modularity of all elliptic curves over all real quadratic fields.

Theorem 1. Let $E$ be an elliptic curve over a real quadratic field $K$. Then $E$ is modular.

Before sketching the strategy of the proof of Theorem 1, we need a little more notation and terminology. Let $p$ be a rational prime. Denote by

$$
\bar{\rho}_{E, p}: G_{K} \rightarrow \operatorname{Aut}(E[p]) \cong \mathrm{GL}_{2}\left(\mathbb{F}_{p}\right)
$$


the representation giving the action of $G_{K}$ on the $p$-torsion of $E$. We say $\bar{\rho}_{E, p}$ is modular if there exists a Hilbert cuspidal eigenform $\mathfrak{f}$ over $K$ of parallel weight 2, and a place $\lambda \mid p$ of $\overline{\mathbb{Q}}$ such that $\bar{\rho}_{E, p}^{\mathrm{ss}} \sim \bar{\rho}_{\mathrm{f}, \lambda}^{\mathrm{ss}}$.

For our purposes, the following is a convenient sketch of the main steps in the proof $([83,[81,[24,[17,[9])$ of modularity of elliptic curves over $\mathbb{Q}$ :

Step I (modularity lifting): one shows that if $\bar{\rho}_{E, p}$ is modular and the image of $\bar{\rho}_{E, p}$ is 'sufficiently large' (in a suitable sense), then $\rho_{E, p}$ is modular.

Step II (Langlands-Tunnell): if $\bar{\rho}_{E, 3}$ is irreducible then it is modular.

Step III (3-5 modularity switching): with the help of Steps I and II, one shows that if $\bar{\rho}_{E, 5}$ is irreducible then it is modular.

Step IV (dealing with exceptions): putting Steps I, II, III together, one sees that $E$ is modular except possibly if the image of $\bar{\rho}_{E, p}$ is 'small' for $p=3$ and 5 simultaneously. Such elliptic curves have certain mod 3 and mod 5 level structures, and so give rise to rational points on certain modular curves. By enumerating these rational points (of which there are only a handful) one shows that these elliptic curves are modular by some other means.

We are ready to outline the proof of Theorem 1 and its similarities and differences from the above; we also outline the contents of the paper. First we need a suitable modularity lifting theorem (Step I), and the following is a relatively straightforward consequence of a theorem of Breuil and Diamond [10, Théorème 3.2.2], which in turn is a consequence of deep results due to Kisin [46], Gee [36], and Barnet-Lamb, Gee and Geraghty [3], 4].

Theorem 2. Let $E$ be an elliptic curve over a totally real number field $K$, and let $p \neq 2$ be a rational prime. Write $\bar{\rho}=\bar{\rho}_{E, p}$. Suppose

(i) $\bar{\rho}$ is modular,

(ii) $\bar{\rho}\left(G_{K\left(\zeta_{p}\right)}\right)$ is absolutely irreducible.

Then $E$ is modular.

The derivation of Theorem 2 from the aforementioned theorem of Breuil and Diamond is given in Section 2. Sections 3 and 4 are devoted to background results on modular curves and images $\bar{\rho}\left(G_{K\left(\zeta_{p}\right)}\right)$ that are needed in the remainder of the paper. In Sections 5 and 6 we explain how Langlands-Tunnell (Step II) and Wiles' 3-5 modularity switching argument (Step III) carry over to the setting of elliptic curves over totally real fields. These, together with Theorem 2 give the following.

Theorem 3. Let $p=3$ or 5 . Let $E$ be an elliptic curve over a totally real field $K$. Suppose that $\bar{\rho}_{E, p}\left(G_{K\left(\zeta_{p}\right)}\right)$ is absolutely irreducible. Then $E$ is modular.

We are interested in proving modularity of elliptic curves over real quadratic fields. If we are to follow the same strategy as over $\mathbb{Q}$, we will have to examine the non-cuspidal real quadratic points on certain modular curves; these parametrize elliptic curves where $\bar{\rho}_{E, 3}\left(G_{K\left(\zeta_{3}\right)}\right)$ and $\bar{\rho}_{E, 5}\left(G_{K\left(\zeta_{5}\right)}\right)$ are simultaneously absolutely reducible. Some of these modular curves (for example the elliptic curve $X_{0}(15)$ ) have infinitely many real quadratic points, and there seems to be no simple way to prove that these points are modular. We are forced to interpolate a further step into the above strategy, by adding a 3-7 modularity switching argument (Section 7), which incorporates some ideas of Manoharmayum [51, [52. 
Theorem 4. Let $E$ be an elliptic curve over a totally real field $K$. Suppose that $\bar{\rho}_{E, 7}\left(G_{K\left(\zeta_{7}\right)}\right)$ is absolutely irreducible. Then $E$ is modular.

Thanks to the addition of Theorem 4, an elliptic curve $E$ over a totally real field $K$ is modular except possibly if the images $\bar{\rho}_{E, p}\left(G_{K\left(\zeta_{p}\right)}\right)$ are simultaneously absolutely reducible for $p=3,5,7$. In Section 8 we show that such an elliptic curve gives rise to a $K$-point on one of 27 modular curves:

$$
X(u, v, w) \quad u \in\{\mathrm{b} 3, \mathrm{~s} 3, \mathrm{~ns} 3\}, \quad v \in\{\mathrm{b} 5, \mathrm{~s} 5, \mathrm{~ns} 5\}, \quad w \in\{\mathrm{b} 7, \mathrm{~s} 7, \mathrm{~ns} 7\} .
$$

See Section 3 for an explanation of the notation, but for now $b, s$ and ns respectively stand for 'Borel', 'normalizer of split Cartan' and 'normalizer of non-split Cartan'. The curves in (11) all have genera $>1$, and so by Faltings' Theorem [29] possess only finitely many $K$-points.

Theorem 5. Let $K$ be a totally real field. All but finitely many $\bar{K}$-isomorphism classes of elliptic curves over $K$ are modular.

Now a possible route to proving Theorem 1 is to attempt to enumerate all noncuspidal real quadratic points on the 27 modular curves in (11) (or suitable quotients) and then prove that the resulting points are modular. This appears to be a monumental task, and some of the curves on this list have defeated us. In Sections 9 and 10 we carry out a more precise examination of cases where $\bar{\rho}_{E, p}\left(G_{K\left(\zeta_{p}\right)}\right)$ is absolutely reducible, and the corresponding modular curves. This allows us (Section 11) to reduce the proof of Theorem 1 to establishing lemmas 1.1 and 1.2 below.

Lemma 1.1. Elliptic curves over $\mathbb{Q}(\sqrt{5})$ are modular.

Lemma 1.2. All non-cuspidal real quadratic points on the modular curves

$$
\begin{gathered}
X(\mathrm{~b} 5, \mathrm{~b} 7), \quad X(\mathrm{~b} 3, \mathrm{~s} 5), \quad X(\mathrm{~s} 3, \mathrm{~s} 5), \\
X(\mathrm{~b} 3, \mathrm{~b} 5, \mathrm{~d} 7), \quad X(\mathrm{~s} 3, \mathrm{~b} 5, \mathrm{~d} 7), \quad X(\mathrm{~b} 3, \mathrm{~b} 5, \mathrm{e} 7), \quad X(\mathrm{~s} 3, \mathrm{~b} 5, \mathrm{e} 7),
\end{gathered}
$$

correspond to modular elliptic curves.

The notation d7 and e7 is explained in Section 10, but for now we remark that they indicate mod 7 level structures that are respectively finer than 'normalizer of split Cartan' and 'normalizer of non-split Cartan'.

The seven modular curves in (2), (3) have genera 3, 3, 4, 97, 153, 73 and 113 . It seems that these modular curves are not amenable to the methods of Mazur [54, Kamienny [42 and Merel [55] (we comment on this at the end of Section 11). In the literature there are explicit methods which, in certain favourable circumstances, are capable of determining all quadratic points on a given curve (e.g. 69, 11]), using an explicit model for the curve and knowledge of the Mordell-Weil group of its Jacobian. As far as we are aware, such methods have only been applied to curves of genus 2 and 3, and our computations for the three curves in (2) pushes these methods to their limit. In Sections 12, 13, 14 we determine the quadratic points on the three modular curves in (2), and deduce modularity of the non-cuspidal real quadratic points.

The four modular curves in (3) are extremely complicated on account of their large genera, and certainly beyond the aforementioned explicit methods found in the literature. Instead we give a representation of these curves as normalizations of fibre products of curves (Section [15), and develop an analogue of the Mordell-Weil sieve that applies to such fibre products (Section 16). Remarkably, we are able to 
show (Section 17) with the help of this sieve, that the non-cuspidal real quadratic points on these curves have $j$-invariants belonging to $\mathbb{Q}$, and so their modularity follows from the modularity of elliptic curves over $\mathbb{Q}$.

The proof Theorem 1 is completed in Section 18 by proving Lemma 1.1. This requires the computation of $\mathbb{Q}(\sqrt{5})$-points on another four modular curves.

We end the paper by briefly mentioning some further implications of this work for modularity of elliptic curves over arbitrary totally real fields (Section [19), and also possible Diophantine applications, including the Fermat equation (Section 20).

Acknowledgements. We would like to thank the referees for useful comments. It is a pleasure to express our sincere gratitude to a large number of colleagues for their help and advice during the course of writing this paper: Samuele Anni, Alex Bartel, Peter Bruin, Frank Calegari, Tommaso Centeleghe, John Cremona, Lassina Dembélé, Fred Diamond, Luis Dieulefait, Toby Gee, Ariel Pacetti, Richard Taylor and Damiano Testa. We would also like to thank Rajender Adibhatla, Shuvra Gupta, Derek Holt, David Loeffler and Panagiotis Tsaknias for useful discussions.

Computational Details. Some of the computational details in Sections 12,18 were carried out in the computer algebra system Magma 7. The reader can find the Magma scripts for verifying these computations (together with extensive commentary) at:

http://arxiv.org/abs/1310.7088

Our programs make use of several Magma packages. We would like to acknowledge these packages and their authors and main contributers:

- Hyperelliptic Curves: Nils Bruin, Brendan Creutz, Steve Donnelly, Michael Harrison, David Kohel, Michael Stoll, Paul van Wamelen;

- Small Modular Curves Database: Michael Harrison;

- Algebraic Function Fields: Florian Heß, Claus Fieker, Nicole Sutherland;

- Modular Forms: William Stein, Kevin Buzzard, Steve Donnelly;

- Modular Abelian Varieties: William Stein, Jordi Quer;

- Matrix Groups over Finite Fields: Eamonn O'Brien.

\section{Part 2. Background}

\section{Modularity Lifting: Proof of Theorem 2}

We assume the hypotheses of Theorem 2 . Thus $K$ is a totally real field, $E$ is an elliptic curve over $K$, and $p \neq 2$ is a rational prime so that $\bar{\rho}:=\bar{\rho}_{E, p}$ is modular, and $\bar{\rho}\left(G_{K\left(\zeta_{p}\right)}\right)$ is absolutely irreducible.

Write $\rho: G_{K} \rightarrow \mathrm{GL}_{2}\left(\mathbb{Z}_{p}\right)$ for the representation arising from the action of $G_{K}$ on the Tate module $T_{p}(E)$. For any finite place $v$ of $K$, we write $D_{v} \subset G_{K}$ for the corresponding decomposition group. We apply Théorème 3.2 .2 of [10] to $\bar{\rho}$. That theorem involves making certain choices and we merely make the obvious ones as follows. We let $S$ be the set of finite places $v$ of $K$ of bad reduction for $E$ together with the places above $p$. We let $T$ be the subset of places above $p$ where $E$ has potentially ordinary reduction or potentially multiplicative reduction. In the notation of that theorem $\epsilon: G_{K} \rightarrow \mathbb{Z}_{p}^{*}$ is the $p$-adic cyclotomic character, and we choose $\psi=\epsilon$. We let $\rho_{v}=\left.\rho\right|_{D_{v}}$. For a place $v$ we write $\left[r_{v}, N_{v}\right]$ for the Weil-Deligne type of $\rho_{v}$. We note ([61, Sections 14 and 15]) that $N_{v}=0$ if and only 
if $E$ has potentially good reduction at $v$. If $p \neq 5$, the conditions of [10, Théorème 3.2.2] are satisfied; that theorem asserts existence of a global modular lift of $\bar{\rho}$ having the same local properties as $\rho$ (properties (i)-(v) in the conclusion of the theorem). It also asserts that every global lift having these properties is modular, so $\rho$ is modular. When $p=5$, the theorem has an extra hypothesis: namely that the projective image of $\left.\bar{\rho}\right|_{G_{K\left(\zeta_{5}\right)}}$ is not $\operatorname{PSL}_{2}\left(\mathbb{F}_{5}\right)$. The reason for this additional hypothesis is to assure the existence of Taylor-Wiles systems (as in [46, Section 3.2.3]). However, in our situation, because $\operatorname{det} \bar{\rho}$ is the $\bmod 5$ cyclotomic character, we can still choose Taylor-Wiles systems without this extra condition, as we now explain. In the notation of [46, Section 3.2.3] (except we change the name of the field to $K$ ) and following the proof of Theorem 2.49 in [23, what we need to show is that for each positive integer $n$ and non-trivial cocycle $\psi \in \mathrm{H}^{1}\left(G_{K, S}, \operatorname{ad}^{0} \bar{\rho}(1)\right)$, we can find a place $v \notin S$ of $K$ such that

(i) $|k(v)| \equiv 1\left(\bmod p^{n}\right)$, and $\bar{\rho}\left(\operatorname{Frob}_{v}\right)$ has distinct eigenvalues.

(ii) The image of $\psi$ under the restriction map

$$
\mathrm{H}^{1}\left(G_{K}, \operatorname{ad}^{0} \bar{\rho}(1)\right) \rightarrow \mathrm{H}^{1}\left(D_{v}, \operatorname{ad}^{0} \bar{\rho}(1)\right)
$$

is non-trivial.

Let $K_{m}$ be the extension of $K\left(\zeta_{p^{m}}\right)$ cut out by $\operatorname{ad}^{0} \bar{\rho}$. The argument in 23, Theorem $2.49]$ then works once we know the restriction of $\psi$ to $\mathrm{H}^{1}\left(G_{K_{0}}, \operatorname{ad}^{0} \bar{\rho}(1)\right)$ is nontrivial. To do this we want to show that $\mathrm{H}^{1}\left(\operatorname{Gal}\left(K_{0} / K\right), \operatorname{ad}^{0} \bar{\rho}(1)^{G_{K_{0}}}\right)=0$. Because $G_{K_{0}}$ acts trivially on $\operatorname{ad}^{0} \bar{\rho}$, the coefficient module vanishes unless $\zeta_{p} \in K_{0}$. Because $\mathrm{H}^{1}\left(\mathrm{PSL}_{2}\left(\mathbb{F}_{5}\right), \operatorname{Sym}^{2} \mathbb{F}_{5}^{2}\right)=\mathbb{F}_{5}$ does not vanish, the extra condition when $p=5$ was used to exclude the possibility that $\operatorname{Gal}\left(K_{0} / K\right)=\mathrm{PSL}_{2}\left(\mathbb{F}_{5}\right)$ and $\zeta_{5} \in K_{0}$. But using the fact that $\bar{\rho}$ has cyclotomic determinant, we can get rid of it as follows. Suppose the above happens, then $\sqrt{5} \in K$ because det $: \bar{\rho}\left(G_{K}\right) \rightarrow \mathrm{PGL}_{2}\left(\mathbb{F}_{5}\right) \rightarrow \mathbb{F}_{5}^{*} /\left(\mathbb{F}_{5}^{*}\right)^{2}$ is induced by the cyclotomic character. But $\zeta_{5} \in K_{0} \backslash K$ implies that $\operatorname{Gal}\left(K_{0} / K\right)$ has a quotient of order 2 . As $\operatorname{Gal}\left(K_{0} / K\right) \cong \mathrm{PSL}_{2}\left(\mathbb{F}_{5}\right) \cong A_{5}$, we have a contradiction.

\section{Background on Modular Curves}

In order to apply Theorem 2, we need a better understanding of its condition (ii). We will show that if condition (ii) fails, then the image of $\bar{\rho}_{E, p}$ lies either in a Borel subgroup of $\mathrm{GL}_{2}\left(\mathbb{F}_{p}\right)$, or in certain subgroups contained in the normalizers of Cartan subgroups. In this case, the elliptic curve $E / K$ gives rise to a noncuspidal $K$-point on a modular curve corresponding to one of these subgroups. We now briefly sketch the relationship between certain subgroups of $\mathrm{GL}_{2}\left(\mathbb{F}_{p}\right)$ and the corresponding modular curves. For more details we recommend the articles by Mazur [53, and by Rohrlich 62.

Let $p$ be a prime, and $H$ a subgroup of $\mathrm{GL}_{2}\left(\mathbb{F}_{p}\right)$ satisfying the condition $-I \in H$. Write $H_{0}=H \cap \mathrm{SL}_{2}\left(\mathbb{F}_{p}\right)$ and denote by $\widetilde{H}$ the preimage of $H_{0}$ via the natural map $\mathrm{SL}_{2}(\mathbb{Z}) \rightarrow \mathrm{SL}_{2}\left(\mathbb{F}_{p}\right)$. Then $\tilde{H}$ is a congruence subgroup of $\mathrm{SL}_{2}(\mathbb{Z})$ of level $p$ and we define the modular curve corresponding to $H$ by

$$
X(H):=\widetilde{H} \backslash \mathfrak{H}^{*}, \quad \mathfrak{H}^{*}:=\mathfrak{H} \cup \mathbb{Q} \cup\{\infty\}
$$

where $\mathfrak{H}$ is upper half-plane. The above construction gives $X(H)$ as compact Riemann surface, or equivalently as an algebraic curve over $\mathbb{C}$. It turns out however that $X(H)$ is defined over the field $\mathbb{Q}\left(\zeta_{p}\right)^{\operatorname{det}(H)}$; here we view $\operatorname{det}(H)$ as subgroup 
of $\operatorname{Gal}\left(\mathbb{Q}\left(\zeta_{p}\right) / \mathbb{Q}\right)$ via the cyclotomic character $\chi_{p}: \operatorname{Gal}\left(\mathbb{Q}\left(\zeta_{p}\right) / \mathbb{Q}\right) \stackrel{\cong}{\rightarrow} \mathbb{F}_{p}^{*}$. We shall assume henceforth that $\operatorname{det}(H)=\mathbb{F}_{p}^{*}$, and so $X(H)$ is defined over $\mathbb{Q}$. The curve $X(H)$ admits a natural map

$$
j: X(H) \rightarrow X(1), \quad\left(X(1):=\mathrm{SL}_{2}(\mathbb{Z}) \backslash \mathfrak{H}^{*} \cong \mathbb{P}^{1}\right)
$$

corresponding to the inclusion $\tilde{H} \subset \mathrm{SL}_{2}(\mathbb{Z})$. The map $j$ is defined over $\mathbb{Q}$, and is ramified only above 0,1728 and $\infty$. We refer to the points of the fibre $j^{-1}(\infty) \subset$ $X(H)(\overline{\mathbb{Q}})$ as the cusps of $X(H)$. For a number field $K$, the non-cuspidal $K$-points of $X(H)$ correspond to $\overline{\mathbb{Q}}$-isomorphism classes of pairs $(E, \eta \circ H)$ of elliptic curves $E / K$ with an $H$-orbit of isomorphisms

$$
\eta: \mathbb{F}_{p}^{2} \stackrel{\cong}{\longrightarrow} E[p](\overline{\mathbb{Q}})
$$

such that $\eta^{-1} \sigma \eta \in H$ for all $\sigma \in G_{K}$ (note that $H$ acts on the set of such $\eta$ by its action on the domain of $\eta$ ). An elliptic curve $E$ over $K$ supports a non-cuspidal point on $X(H)$ if and only if the image $\bar{\rho}_{E, p}\left(G_{K}\right)$ is conjugate to a subgroup of $H$. In general, it is possible for one $E$ to support distinct points of $X(H)$. In fact, picking an $\eta$, the number of points supported on $E$ is the number of $G_{K}$-fixed points of the double coset space

$$
\eta^{-1} \cdot \operatorname{Aut}_{\overline{\mathbb{Q}}}(E) \cdot \eta \backslash \mathrm{GL}_{2}\left(\mathbb{F}_{p}\right) / H,
$$

where the action of $G_{K}$ depends on the chosen $\eta$. Moreover if $E / K$ gives rise to the $K$-point $P$ on $X(H)$ then $j(P)$ is the $j$-invariant of $E$. If two elliptic curves $E_{1}$, $E_{2}$ defined over $K$, and having $j$-invariants $\neq 0,1728$ give rise to the same $K$-point of $X(H)$, then they have the same $j$-invariant and hence are quadratic twists of each other.

3.1. Borel Subgroups, Cartan Subgroups, and Normalizers. Let $p \geq 3$ be a prime. Let $B(p)$ denote the Borel subgroup of $\mathrm{GL}_{2}\left(\mathbb{F}_{p}\right)$. Let $C_{\mathrm{S}}(p)$ and $C_{\mathrm{ns}}(p)$ respectively denote the split and non-split Cartan subgroups of $\mathrm{GL}_{2}\left(\mathbb{F}_{p}\right)$, and let $C_{\mathrm{s}}^{+}(p)$ and $C_{\mathrm{ns}}^{+}(p)$ respectively be their normalizers. The three groups $B(p), C_{\mathrm{s}}^{+}(p)$ and $C_{\mathrm{ns}}^{+}(p)$ contain $-I$ and have determinants equal to $\mathbb{F}_{p}^{*}$. Thus they correspond to modular curves defined over $\mathbb{Q}$. The usual notation for these modular curves is $X_{0}(p), X_{\text {split }}(p)$ and $X_{\text {nonsplit }}(p)$. We shall find it convenient to denote these modular curves by the following non-standard notation: $X(\mathrm{~b} p), X(\mathrm{~s} p)$ and $X(\mathrm{~ns} p)$, so for example $X_{0}(5), X_{\text {split }}(5)$ and $X_{\text {nonsplit }}(5)$ become $X(\mathrm{~b} 5), X(\mathrm{~s} 5), X(\mathrm{~ns} 5)$.

3.2. Fibre Products. Now let $p_{1}, \ldots, p_{r} \geq 3$ be distinct primes. Let $H_{i} \subseteq \mathrm{GL}_{2}\left(\mathbb{F}_{p_{i}}\right)$ be subgroups, all satisfying $-I \in H_{i}$ and $\operatorname{det}\left(H_{i}\right)=\mathbb{F}_{p_{i}}^{*}$. Let $\widetilde{H_{i}}$ be the corresponding congruence subgroups of $\mathrm{SL}_{2}(\mathbb{Z})$ defined above. Then $\cap \widetilde{H}_{i}$ is a congruence subgroup of $\mathrm{SL}_{2}(\mathbb{Z})$ of level $\prod p_{i}$. We define $X\left(H_{1}, \ldots, H_{r}\right)$ to be the modular curve

$$
X\left(H_{1}, \ldots, H_{r}\right):=\left(\widetilde{H_{1}} \cap \cdots \cap \widetilde{H_{r}}\right) \backslash \mathfrak{H}^{*} .
$$

This again is defined over $\mathbb{Q}$, and is the normalization of the fibre product

$$
X\left(H_{1}\right) \times_{X(1)} X\left(H_{2}\right) \times_{X(1)} \cdots \times_{X(1)} X\left(H_{r}\right) .
$$

Again $X\left(H_{1}, \ldots, H_{r}\right)$ is naturally endowed with a $j$-map to $X(1)$ coming from the inclusion $\widetilde{H_{1}} \cap \cdots \cap \widetilde{H_{r}} \subseteq \mathrm{SL}_{2}(\mathbb{Z})$. This agrees with the composition of the projection onto any of the $X\left(H_{i}\right)$ with $j: X\left(H_{i}\right) \rightarrow X(1)$. If $K$ is a number field, non-cuspidal 
$K$-points correspond to (isomorphism classes of) pairs ( $\left.E, \eta \circ \prod H_{i}\right)$ of elliptic curves $E / K$ with an $\prod H_{i}$-orbit of isomorphisms

$$
\eta: \prod \mathbb{F}_{p_{i}}^{2} \stackrel{\cong}{\longrightarrow} E\left[\prod p_{i}\right](\overline{\mathbb{Q}})
$$

such that $\eta^{-1} \bar{\rho}_{E, p_{i}}\left(G_{K}\right) \eta$ is contained in $H_{i}$, for $i=1, \ldots, r$.

In the notation $X\left(H_{1}, \ldots, H_{r}\right)$, we shall replace the group $H_{i}$ with $\mathrm{b} p$, sp, nsp if it equals $B_{0}(p), C_{\mathrm{s}}^{+}(p)$ or $C_{\mathrm{ns}}^{+}(p)$ respectively. Thus for example, $X(\mathrm{~b} 5, \mathrm{~b} 7)$ is isomorphic to $X(\mathrm{~b} 5) \times_{X(1)} X(\mathrm{~b} 7)$ and in standard notation can be denoted by $X_{0}(35)$.

\section{The Image $\bar{\rho}_{E, p}\left(G_{K\left(\zeta_{p}\right)}\right)$}

The following proposition is our first attempt at understanding condition (ii) of Theorem 2, It relates the failure of condition (ii) to $K$-points on the modular curves $X(\mathrm{~b} p), X(\mathrm{~s} p)$ and $X(\mathrm{~ns} p)$.

Proposition 4.1. Let $E$ be an elliptic curve over a totally real field $K$. Let $p \geq 3$ be a rational prime, and write $\bar{\rho}=\bar{\rho}_{E, p}$. Then

(i) $\bar{\rho}\left(G_{K\left(\zeta_{p}\right)}\right)=\bar{\rho}\left(G_{K}\right) \cap \mathrm{SL}_{2}\left(\mathbb{F}_{p}\right)$.

(ii) If $\bar{\rho}\left(G_{K\left(\zeta_{p}\right)}\right)$ is absolutely reducible, then $\bar{\rho}\left(G_{K}\right)$ is contained either in a Borel subgroup, or in the normalizer of a Cartan subgroup. In this case $E$ gives rise to a non-cuspidal $K$-point on $X(\mathrm{~b} p), X(\mathrm{~s} p)$ or $X(\mathrm{~ns} p)$.

Proof. The determinant of $\bar{\rho}$ is the mod $p$ cyclotomic character

$$
\chi_{p}: G_{K} \rightarrow \operatorname{Aut}\left(\mu_{p}\right) \cong \mathbb{F}_{p}^{*},
$$

where $\mu_{p}$ is the group of $p$-th roots of unity. The first part follows immediately.

Write $G=\bar{\rho}\left(G_{K}\right)$, and suppose $G$ is not contained in a Borel subgroup. Then $G$ is irreducible, and as $K$ is totally real it is absolutely irreducible. The second part follows from Lemma 4.2 below.

Lemma 4.2. Let $p \geq 3$ be prime. Let $G$ be an absolutely irreducible subgroup of $\mathrm{GL}_{2}\left(\mathbb{F}_{p}\right)$ such that $G \cap \mathrm{SL}_{2}\left(\mathbb{F}_{p}\right)$ is absolutely reducible. Then $G$ is contained in the normalizer of a Cartan subgroup.

If moreover $\operatorname{det}(G)=\mathbb{F}_{p}^{*}$, then $G \cap \mathrm{GL}_{2}^{+}\left(\mathbb{F}_{p}\right)$ is contained in a Cartan subgroup, where $\mathrm{GL}_{2}^{+}\left(\mathbb{F}_{p}\right)$ is the subgroup of $\mathrm{GL}_{2}\left(\mathbb{F}_{p}\right)$ consisting of matrices with square determinant.

Proof. Write $G^{\prime}=G \cap \mathrm{SL}_{2}\left(\mathbb{F}_{p}\right)$. If $p$ divides the order of $G$ then (e.g. [64, Section 2] or [78, Lemma 2]) either $G$ contains $\mathrm{SL}_{2}\left(\mathbb{F}_{p}\right)$ or $G$ is contained in a Borel subgroup. In the former case, $G^{\prime}$ contains $\mathrm{SL}_{2}\left(\mathbb{F}_{p}\right)$ and so is absolutely irreducible, and in the latter case, $G$ is reducible. We may thus suppose that $p$ does not divide the order of $G$.

Write $H$ for the image of $G$ in $\mathrm{PGL}_{2}\left(\mathbb{F}_{p}\right)$, and let $n$ be its order. Let $\mathcal{M}$ be the set of points $P \in \mathbb{P}^{1}\left(\overline{\mathbb{F}}_{p}\right)$ that are fixed by at least one non-trivial element of $H$. Swinnerton-Dyer shows [78, pages 13-14] that $\mathcal{M}$ is stable under the action of $G$, and that it can be written as the union of disjoint orbits $\mathcal{N}_{1}, \ldots, \mathcal{N}_{\nu}$, with the following exhaustive list of possibilities.

(a) $H$ is cyclic, $G$ is contained in a Cartan subgroup: $\nu=2,\left|\mathcal{N}_{1}\right|=\left|\mathcal{N}_{2}\right|=1$;

(b) $H$ is dihedral, $G$ is contained in the normalizer of a Cartan subgroup: $\nu=3$, $n \geq 4$ even, $\left|\mathcal{N}_{1}\right|=\left|\mathcal{N}_{2}\right|=n / 2,\left|\mathcal{N}_{3}\right|=2 ;$ 
(c) $H$ is isomorphic to $A_{4}: \nu=3, n=12,\left|\mathcal{N}_{1}\right|=6,\left|\mathcal{N}_{2}\right|=\left|\mathcal{N}_{3}\right|=4$;

(d) $H$ is isomorphic to $S_{4}: \nu=3, n=24,\left|\mathcal{N}_{1}\right|=12,\left|\mathcal{N}_{2}\right|=8,\left|\mathcal{N}_{3}\right|=6$;

(e) $H$ is isomorphic to $A_{5}: \nu=3, n=60,\left|\mathcal{N}_{1}\right|=30,\left|\mathcal{N}_{2}\right|=20,\left|\mathcal{N}_{3}\right|=12$.

In our situation, case (a) is easy to rule out as it forces $G$ to be absolutely reducible -indeed, the unique elements of $\mathcal{N}_{1}, \mathcal{N}_{2}$ are fixed by $G$. We would like to rule out cases (c), (d), (e).

Observe that $G^{\prime}$ is the kernel of the restriction to $G$ of $\operatorname{det}: G \rightarrow \mathbb{F}_{p}^{*}$. Thus $G^{\prime}$ is normal in $G$ with cyclic quotient. If $G^{\prime}$ consists only of scalar matrices, then $H$ is a quotient of $G / G^{\prime}$ and so is cyclic, which contradicts possibilities (b)-(e). Thus $G^{\prime}$ contains some non-scalar matrix $g$. The image of $g$ in $H$ is non-trivial.

As $G^{\prime}$ is absolutely reducible, there is some $P \in \mathbb{P}^{1}\left(\overline{\mathbb{F}}_{p}\right)$ which is fixed by $G^{\prime}$. In particular, $P$ belongs to one of the orbits $\mathcal{N}_{i}$, which we denote by $\mathcal{N}$. If $Q$ is some other element of $\mathcal{N}$ then its stabilizer in $G$ is conjugate to the stabilizer of $P$. However, $G^{\prime}$ is normal in $G$ and is contained in the stabilizer of $P$. Thus $G^{\prime}$ stabilizes $Q$ too. It follows that every element $Q$ of $\mathcal{N}$ is an eigenvector of $g$. However, $g$ is a non-scalar and $p$ does not divide its order (as $g \in G$ ). Thus $g$ has precisely two eigenvectors in $\mathbb{P}^{1}\left(\overline{\mathbb{F}}_{p}\right)$. Hence $|\mathcal{N}| \leq 2$. From the above list of cases (b)-(e), we see that we are in case (b), and $|\mathcal{N}|=2$. This proves the first part of the lemma.

For the second part, suppose that $\operatorname{det}(G)=\mathbb{F}_{p}^{*}$. Let $G^{+}$be the stabilizer of $P$, which by the above contains $G^{\prime}$. Since $G$ acts transitively on $\mathcal{N}$, which has size 2, the subgroup $G^{+}$must have index 2. From the isomorphism det $: G / G^{\prime} \rightarrow \mathbb{F}_{p}^{*}$ we see that $\operatorname{det}\left(G^{+} / G\right)$ consists of the squares in $\mathbb{F}_{p}^{*}$. Hence $G^{+}=G \cap \mathrm{GL}_{2}^{+}\left(\mathbb{F}_{p}\right)$. Now $G^{+}$stabilizes $P$, and by the argument above also stabilizes the other element of $\mathcal{N}$. Thus $G^{+}$is contained in a Cartan subgroup.

\section{Langlands-Tunnell: Proof of Theorem 3 for $p=3$}

We shall need the following consequence of the well-known Langlands-Tunnell Theorem.

Theorem 6 (Langlands-Tunnell). Let $E$ be an elliptic curve over a totally real number field $K$ and suppose $\bar{\rho}_{E, 3}$ is irreducible. Then $\bar{\rho}_{E, 3}$ is modular.

The deduction of Theorem 6 from the Langlands-Tunnell Theorem [48, 82] is well-known; a proof is given by Dieulefait and Freitas in [26, Lemma 4.2] and follows the lines of the argument by Wiles [83. Chapter 5] for classical modular forms.

The following is an immediate corollary of Langlands-Tunnell and Theorem 2 It is of course Theorem 3 with $p=3$.

Corollary 5.1. If $E$ is an elliptic curve over a totally real field $K$ and $\bar{\rho}_{E, 3}\left(G_{K\left(\zeta_{3}\right)}\right)$ is absolutely irreducible, then $E$ is modular.

\section{Part 3. Modularity Switching}

\section{3-5 Modularity Switching: Proof of Theorem 3 for $p=5$}

In this section we prove Theorem 3 for $p=5$. This proof follows from Wiles' original 3-5 modularity switching argument over $\mathbb{Q}$ [83, Proof of Theorem 5.2]. For the convenience of the reader and as a warm-up for the more elaborate switching argument in the next section, we include a sketch. 
Lemma 6.1. Let $E$ be an elliptic curve over a number field $K$. Then there is an elliptic curve $E^{\prime} / K$ such that

(i) $\bar{\rho}_{E, 5} \sim \bar{\rho}_{E^{\prime}, 5}$;

(ii) $\bar{\rho}_{E^{\prime}, 3}\left(G_{K}\right)$ contains $\mathrm{SL}_{2}\left(\mathbb{F}_{3}\right)$.

We first show how the lemma implies Theorem 3 for $p=5$.

Proof of Theorem 3 for $p=5$. Let $E$ be an elliptic curve over a totally real number field $K$ and let $E^{\prime}$ be as in the statement of Lemma6.1. It follows from Corollary5.1 that $E^{\prime}$ is modular. Thus $\bar{\rho}_{E, 5} \sim \bar{\rho}_{E^{\prime}, 5}$ is modular. Applying Theorem 2 to $E$ with $p=5$, we see that $E$ is modular.

Let us now prove lemma 6.1 Let $K$ be a number field and $E / K$ is an elliptic curve. Let us denote by $X_{E}(5)$ the twisted modular curve over $K$ which classifies elliptic curves $E^{\prime}$ with a symplectic isomorphism of group schemes $E[5] \rightarrow E^{\prime}[5]$. Here the adjective symplectic means that the isomorphism is compatible with the Weil pairings on $E[5]$ and $E^{\prime}[5]$. We observe the following properties

- $X_{E}(5) \cong \mathbb{P}^{1}$ over $K$. This is because $X_{E}(5)$ isomorphic (over $\bar{K}$ ) to the standard modular curve $X(5)$, and hence is a smooth curve of genus 0 over $K$, possessing by construction a $K$-rational point (coming from $E$ itself).

- There is bijection between the non-cuspidal $K$-points on $X_{E}(5)$ and classes of pairs $\left(E^{\prime}, u\right)$ where $E^{\prime}$ is an elliptic curve over $K$ and $u$ is a symplectic isomorphism $E[5] \rightarrow E^{\prime}[5]$ of $G_{K}$-modules. Two such pairs $\left(E_{1}^{\prime}, u_{1}\right)$ and $\left(E_{2}^{\prime}, u_{2}\right)$ belong to the same class if there is a $K$-isomorphism $\phi: E_{1}^{\prime} \rightarrow E_{2}^{\prime}$ such that $u_{2}=\phi \circ u_{1}$.

Thus a non-cuspidal $K$-point of $X_{E}(5)$ gives an elliptic curve $E^{\prime}$ satisfying condition (i), and we want to find such a point where (ii) holds. The maximal subgroups of $\mathrm{GL}_{2}\left(\mathbb{F}_{3}\right)$ are $\mathrm{SL}_{2}\left(\mathbb{F}_{3}\right)$, the Borel subgroups, and the normalizers of non-split Cartan subgroups 1 . If the image of $\bar{\rho}_{E^{\prime}, 3}$ does not contain $\mathrm{SL}_{2}\left(\mathbb{F}_{3}\right)$ then $E^{\prime}$ gives rise to a $K$ point of $X_{E}(5) \times_{X(1)} X(\mathrm{~b} 3)$ or $X_{E}(5) \times_{X(1)} X(\mathrm{~ns} 3)$. Over $\mathbb{C}$, the normalizations of these two curves are isomorphic to the modular curves $X(5, \mathrm{~b} 3)$ and $X(5, \mathrm{~ns} 3)$, and hence are irreducible. It follows from Hilbert's irreducibility theorem 66, Theorem 3.4.1] that there is a non-cuspidal $K$-rational point of $X_{E}(5)$ which does not lift to a $K$-rational point of $X_{E}(5) \times_{X(1)} X(\mathrm{~b} 3), X_{E}(5) \times_{X(1)} X(\mathrm{~ns} 3)$. This point provides the required $E^{\prime}$.

\section{3-7 Modularity Switching: Proof of Theorem 4}

In this section we prove Theorem 4. The proof resembles Wiles' 3-5 modularity switching argument sketched in the previous section. In fact, similar arguments have been used to prove special cases of Serre's modularity conjecture for $\mathrm{GL}_{2}\left(\mathbb{F}_{q}\right)$ for $q=5,7$ and 9 by Taylor [80], Manoharmayum [51, [52] and Ellenberg [27. Our argument in this section is closest in spirit to Manoharmayum's; however we benefit from having at our disposal a more powerful modularity lifting theorem, allowing us to work in greater generality. We shall need the following lemma.

Lemma 7.1. Let $E$ be an elliptic curve over a totally real number field $K$. Then there is an extension $L / K$ and an elliptic curve $E^{\prime} / L$ such that

\footnotetext{
${ }^{1} \mathrm{In}_{\mathrm{GL}_{2}}\left(\mathbb{F}_{3}\right)$ the normalizer of a split Cartan subgroup is contained as an index 2 subgroup in the normalizer of a non-split Cartan subgroup, as the latter is a 2-Sylow subgroup.
} 
(i) $L$ is totally real;

(ii) $[L: K]=4$;

(iii) $L \cap K(E[7])=K$;

(iv) $\left.\bar{\rho}_{E, 7}\right|_{G_{L}} \sim \bar{\rho}_{E^{\prime}, 7}$

(v) $\bar{\rho}_{E^{\prime}, 3}\left(G_{L}\right)$ contains $\mathrm{SL}_{2}\left(\mathbb{F}_{3}\right)$.

Before proving Lemma 7.1 we use it prove Theorem 4 .

Proof of Theorem 4. Let $E$ be an elliptic curve over a totally real number field $K$ and let $L$ and $E^{\prime}$ be as in the statement of Lemma 7.1. It follows from Corollary 5.1 that $E^{\prime}$ is modular. Thus $\bar{\rho}_{E^{\prime}, 7}$ is modular. Hence $\left.\bar{\rho}_{E, 7}\right|_{G_{L}}$ is modular.

From now on, we shall only be concerned with the elliptic curve $E$, and will write $\bar{\rho}=\bar{\rho}_{E, 7}$. We will shortly show that $\bar{\rho}\left(G_{L\left(\zeta_{7}\right)}\right)$ is absolutely irreducible. Thus we may apply Theorem 2 to deduce that $E / L$ is modular. Now by a repeated application of cyclic base change results of Langlands [48, it follows that $E / K$ is modular.

All that remains to show is that $\bar{\rho}_{E, 7}\left(G_{L\left(\zeta_{7}\right)}\right)$ is absolutely irreducible. It is here that we make use of the hypothesis in Theorem 4 that $\bar{\rho}\left(G_{K\left(\zeta_{7}\right)}\right)$ is absolutely irreducible. By Proposition 4.1,

$$
\bar{\rho}\left(G_{K\left(\zeta_{7}\right)}\right)=\bar{\rho}\left(G_{K}\right) \cap \mathrm{SL}_{2}\left(\mathbb{F}_{7}\right), \quad \bar{\rho}\left(G_{L\left(\zeta_{7}\right)}\right)=\bar{\rho}\left(G_{L}\right) \cap \mathrm{SL}_{2}\left(\mathbb{F}_{7}\right) .
$$

To complete the proof it is enough to show that $\bar{\rho}\left(G_{K}\right)=\bar{\rho}\left(G_{L}\right)$. The former is the Galois group of $K(E[7]) / K$ and the latter is the Galois group of $L(E[7]) / L$. Since $L \cap K(E[7])=K$ by part (iii) of Lemma 7.1 we see that these groups are the same.

We now turn our attention to proving Lemma 7.1 As before $K$ is a totally real number field and $E / K$ is an elliptic curve. We shall need to work with the modular curve $X_{E}(7)$. For detailed expositions of the construction and properties of $X_{E}(7)$ we recommend the paper of Halberstadt and Kraus [37, and that of Poonen, Schaefer and Stoll [58]. For our needs, the following basic facts found in the above references are sufficient.

- $X_{E}(7)$ is a smooth plane quartic curve defined over $K$.

- $X_{E}(7)$ is a twist of the Klein quartic

$$
X(7): x^{3} y+y^{3} z+z^{3} x=0 .
$$

- For any extension $L / K$, there is bijection between the non-cuspidal $L$-points on $X_{E}(7)$ and classes of pairs $\left(E^{\prime}, u\right)$ where $E^{\prime}$ is an elliptic curve over $L$ and $u$ is a symplectic isomorphism $E[7] \rightarrow E^{\prime}[7]$ of $G_{L}$-modules. Two such pairs $\left(E_{1}^{\prime}, u_{1}\right)$ and $\left(E_{2}^{\prime}, u_{2}\right)$ belong to the same class if there is an $L$-isomorphism $\phi: E_{1}^{\prime} \rightarrow E_{2}^{\prime}$ such that $u_{2}=\phi \circ u_{1}$.

An elliptic curve $E^{\prime}$ as in the statement of Lemma 7.1 corresponds to a point on $X_{E}(7)$ over a certain totally real quartic extension $L / K$ satisfying some additional hypotheses. We shall generate points on $X_{E}(7)$ defined over quartic extensions by taking the intersection of $X_{E}(7)$ with suitable $K$-lines. Write $\check{\mathbb{P}}^{2}$ for the dual space of $\mathbb{P}^{2}$ - this is the parameter space of lines in $\mathbb{P}^{2}$, and is in fact isomorphic to $\mathbb{P}^{2}$. To generate a point on $X_{E}(7)$ that satisfies (ii)-(v) we will choose a line $\ell \in \check{\mathbb{P}}^{2}(K)$ that avoids certain thin subsets of $\check{\mathbb{P}}^{2}(K)$. To satisfy (i), in addition to avoiding these thin subsets, we want our line to belong to a certain open subset of $\breve{\mathbb{P}}^{2}\left(K_{\sigma}\right)$ for each embedding $\sigma: K \hookrightarrow \mathbb{R}$. 
It is helpful to recall the notion of thin subsets [65. Chapter 9], 66. Chapter 3]. Let $n \geq 1$. By a thin subset of $\mathbb{P}^{n}(K)$ of type $\mathbf{I}$ we mean a subset contained in $V(K)$ where $V$ is a proper Zariski-closed $K$-subvariety of $\mathbb{P}^{n}$. By a thin subset of $\mathbb{P}^{n}(K)$ of type II we mean a subset contained in $\pi(V(K))$ where $\pi: V \rightarrow \mathbb{P}^{n}$ is a generically surjective $K$-morphism of degree $\geq 2$ from an irreducible variety $V$ of $\operatorname{dimension} \operatorname{dim}(V)=n$. A thin subset of $\mathbb{P}^{n}(K)$ is a subset contained in the finite union of thin subsets of types I and II. Hilbert's Irreducibility Theorem 66, Theorem 3.4.1] asserts that a thin subset cannot equal $\mathbb{P}^{n}(K)$.

Proof of Lemma 7.1. Fix a totally real field $K$ and an elliptic curve $E / K$. Write $X=X_{E}(7) \subset \mathbb{P}^{2}$. We shall write $X^{(n)}$ for the $n$-th symmetric power of $X$ (see for example [56, Section 3]). This is a smooth $n$-dimensional variety over $K$, whose $K$-points correspond to effective degree $n$ divisors on $X$ that are stable under the action of $G_{K}$. Let $\phi$ be the morphism

$$
\phi: \check{\mathbb{P}}^{2} \hookrightarrow X^{(4)}, \quad \ell \mapsto \ell \cdot X
$$

We define certain thin subsets $S_{1}, \ldots, S_{6}$ of $\check{\mathbb{P}}^{2}(K)$ and choose the line to lie outside these subsets. We let $S_{1}$ be the set of lines $\ell \in \check{\mathbb{P}}^{2}(K)$ passing through any point belonging to either of these two finite sets:

(a) $\bar{K}$-cusps of $X_{E}$;

(b) $X_{E}(K(E[7]))$ (which is certainly a finite set by Faltings' Theorem [29]).

The set $S_{1}$ is plainly a union of thin subsets of type I. Let $S_{2}$ be the set of lines $\ell \in \check{\mathbb{P}}^{2}(K)$ that are tangent to $X$. These belong to the dual curve of $X$, and hence $S_{2}$ is a thin subset of type I. Now any line belonging to $\check{\mathbb{P}}^{2}(K) \backslash S_{1} \cup S_{2}$ intersects $X$ either in an irreducible divisor of degree 4 , or in the sum of two irreducible divisors of degree 2 .

Write $\epsilon$ for the natural map

$$
\epsilon: X^{(2)} \times X^{(2)} \rightarrow X^{(4)}, \quad\left(D_{1}, D_{2}\right) \mapsto D_{1}+D_{2} .
$$

We let $S_{3}$ be the set of lines $\ell \in \check{\mathbb{P}}^{2}(K)$ so that $\phi(\ell)$ belongs to $\epsilon\left(X^{(2)}(K) \times X^{(2)}(K)\right)$. The set $S_{3}$ certainly contains all $K$-lines $\ell$ so that $\ell \cdot X$ is the sum of two irreducible divisors of degree 2 . We would like to show that $S_{3}$ is a thin set. It is known for a curve $C$ of genus at least 2 that $C^{(2)}(K)$ is finite, unless $C$ is hyperelliptic or bielliptic [38, Corollary 3]. In our situation $X$ is not hyperelliptic, but might be bielliptic [5, Section 4], [37, Théorème 8.1]. At any rate, $X^{(2)}$ is isomorphic to its image in the Jacobian of $X$ under an Abel-Jacobi map. It follows from a theorem of Faltings [30] that

$$
X^{(2)}(K)=\left\{D_{1}, \ldots, D_{n}\right\} \cup\left\{E_{1}(K), \ldots, E_{m}(K)\right\}
$$

where the $D_{i}$ are finitely many divisors of degree 2 and the $E_{i}$ are finitely many elliptic curves defined over $K$ lying on $X^{(2)}$. Now $\epsilon\left(X^{(2)}(K) \times X^{(2)}(K)\right)$ consists of a union of isolated points $D_{i}+D_{j}$, the $K$-points of curves $D_{i} \times E_{j}$ and the $K$-points of surfaces $E_{i} \times E_{j}$. The former two categories will contribute to $S_{3}$ thin sets of type I. To show that $S_{3}$ is a thin set, it is enough to show for any $i, j$ that there is no $K$-rational map $\psi: \check{\mathbb{P}}^{2} \rightarrow E_{i} \times E_{j}$, birational onto its image, making the following 
diagram commute:

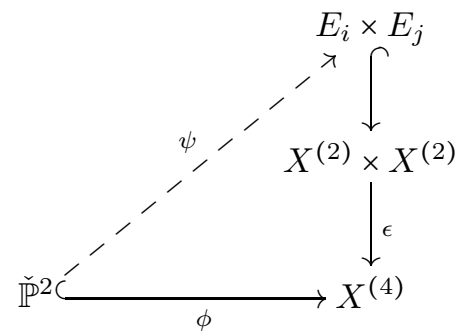

Any $E_{i} \times E_{j}$ is an abelian variety and the image of any rational map $\check{\mathbb{P}}^{2} \rightarrow E_{i} \times E_{j}$ must be constant [56. Corollary 3.9], therefore $S_{3}$ is thin.

Now if $\ell \in \check{\mathbb{P}}^{2}(K) \backslash \cup_{1 \leq i \leq 3} S_{i}$ then $\ell \cdot X$ is an irreducible effective divisor of degree 4 , and so there is some quartic extension $L / K$ and a point $P \in X(L)$ such that $\ell$ passes through $P$. Moreover, $P$ is a non-cusp, and corresponds to a pair $\left(E^{\prime}, u\right)$ where $E^{\prime}$ is an elliptic curve over $L$ and $u: E[7] \rightarrow E^{\prime}[7]$ is a symplectic isomorphism of $G_{L}$-modules. This supplies an extension $L$ and elliptic curve $E^{\prime}$ satisfying (ii) and (iv). To obtain condition (iii), we need to remove another thin set. When (iii) fails, $K(E[7]) \cap L$ is either equal to $L$ or is a quadratic extension $M / K$ contained in $K(E[7])$. The former is impossible by (b) above. To eliminate the latter possibility, note that there are at most finitely many $M \subset K(E[7])$ that are quadratic extensions of $K$, and for each of these we want our line to avoid the image of the natural map

$$
X^{(2)}(M) \rightarrow X^{(4)}(K), \quad D \mapsto D+D^{\sigma},
$$

where $\sigma$ denotes conjugation. Of course we have a decomposition of $X^{(2)}(M)$ similar to (5), where the $M$ replaces $K$, and the elliptic curves $E_{i}$ are defined over $M$. By mimicking the above argument with $E_{i} \times E_{i}^{\sigma}$ replacing $E_{i} \times E_{j}$, we deduce the existence of a thin set $S_{4}$ such that for $\ell \in \check{\mathbb{P}}^{2}$ avoiding $S_{1}, \ldots, S_{4}$, the conditions (ii), (iii) and (iv) hold.

We now look at condition (v). As before, we use the fact that the maximal subgroups of $\mathrm{GL}_{2}\left(\mathbb{F}_{3}\right)$ are $\mathrm{SL}_{2}\left(\mathbb{F}_{3}\right)$, the Borel subgroups, and the normalizers of non-split Cartan subgroups. If the image of $\bar{\rho}_{E^{\prime}, 3}$ does not contain $\mathrm{SL}_{2}\left(\mathbb{F}_{3}\right)$ then $E^{\prime}$ gives rise to an $L$-point on either of the geometrically irreducible curves $Y_{1}$ or $Y_{2}$, which are the normalizations of $X \times_{X(1)} X(\mathrm{~b} 3)$ or $X \times_{X(1)} X(\mathrm{~ns} 3)$ respectively. In particular, $\ell \cdot X$ belongs to the image of $Y^{(4)}(K) \rightarrow X^{(4)}(K)$ where $Y=Y_{1}$ or $Y_{2}$. By Lemma 7.2 below, such $\ell$ respectively belong to thin sets $S_{5}, S_{6}$. Choosing $\ell$ outside $S_{1}, \ldots, S_{6}$ yields an $L$ and $E^{\prime}$ satisfying (ii)-(v).

Finally we want to show that we can choose $\ell$ so that $L$ is totally real. Let $\sigma_{1}, \ldots, \sigma_{n}$ be the real places of $K$. Write $X_{\sigma_{i}} / \mathbb{R}$ for $X \times_{K} K_{\sigma_{i}}$. Then $X_{\sigma_{i}}$ is a real twist of the Klein quartic (44). By Lemma 7.3 below, the $X_{\sigma_{i}}$ are in fact isomorphic to $X(7)$ over $\mathbb{R}$. The line $2 y+z=0$ meets the model given for $X(7)$ in (4) in four distinct real points. Thus there are non-empty open subsets $U_{i} \subset \check{\mathbb{P}}^{2}\left(K_{\sigma_{i}}\right)$ such that any $\ell \in U_{i}$ meets $X_{\sigma_{i}}$ in four real points. Let

$$
W=\check{\mathbb{P}}^{2}(K) \cap \prod_{i=1}^{n} U_{i} .
$$

Now if $\ell \in W \backslash \cup S_{i}$ then the field $L$ is totally real. All that remains is to show that $W$ is not thin, and so the difference $W \backslash \cup S_{i}$ is non-empty. Note that as 
$\prod_{i=1}^{n} \check{\mathbb{P}}^{2}(\mathbb{R})$ is compact, it is covered by finitely many translates of $\prod_{i=1}^{n} U_{i}$ under $\prod_{i=1}^{n} \mathrm{PGL}_{3}(\mathbb{R})$. As $\mathrm{PGL}_{3}(K)$ is dense in $\prod_{i=1}^{n} \mathrm{PGL}_{3}(\mathbb{R})$ by weak approximation, we can assume the translates are given by elements in $\mathrm{PGL}_{3}(K)$. Thus $\check{\mathbb{P}}^{2}(K)$ is the union of finitely many translates of $W$ by the action of $\mathrm{PGL}_{3}(K)$. By Hilbert's irreducibility, $W$ is not thin and so $W \backslash \cup S_{i}$ is non-empty.

Lemma 7.2. Let $X \subset \mathbb{P}^{2}$ be a smooth quartic curve over a number field $K$ and $\mu: Y \rightarrow X$ be $K$-morphism of degree at least 2 . Write $\mu$ also for the induced map $Y^{(4)} \rightarrow X^{(4)}$. Let $\phi: \check{\mathbb{P}}^{2} \rightarrow X^{(4)}$ be the map sending a line $\ell$ to the zero-cycle $\ell \cdot X$. Then the induced map $\mu^{-1}\left(\phi\left(\check{\mathbb{P}}^{2}\right)\right) \rightarrow \phi\left(\check{\mathbb{P}}^{2}\right)$ does not have a rational section.

Proof. By Riemann-Hurwitz, the genus of $Y$ is at least 5. We argue by contradiction. Suppose the map has a rational section. Then we have a commutative triangle,

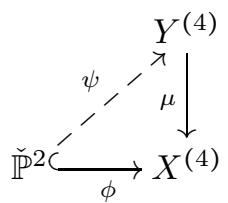

where $\psi$ is a $K$-rational map with $\check{\mathbb{P}}^{2}$ birational to its image. Choose $D \in Y^{(4)}(K)$ belonging to the image of $\check{\mathbb{P}}^{2}(K)$ under $\psi$. Note that $\psi\left(\check{\mathbb{P}}^{2}\right)$ is a rational surface and so its image in the Jacobian $J_{Y}$ of $Y$ under a suitable Abel-Jacobi map $\psi\left(\check{\mathbb{P}}^{2}\right) \subset$ $Y^{(4)} \rightarrow J_{Y}$ must be a single point. Thus the closed points of $\psi\left(\check{\mathbb{P}}^{2}\right)$ (interpreted as divisors on $Y$ ) are linearly equivalent to $D$. It follows that $\mathbb{P}(L(D))$ has dimension at least 2 , and so $l(D) \geq 3$. An easy application of Riemann-Roch shows that $D$ is a special divisor (i.e. $l\left(K_{Y}-D\right)>0$ where $K_{Y}$ is the canonical divisor on $Y$ ). Clifford's Theorem [39, Theorem IV.5.4] states that

$$
l(D)-1 \leq \frac{\operatorname{deg}(D)}{2},
$$

and that if equality holds then $D$ is either canonical, or $Y$ is hyperelliptic. Since $\operatorname{deg}(D)=4$, we see that $l(D)=3$ and so the Clifford inequality (6) is in fact an equality. Thus $D$ is either canonical, or $Y$ is hyperelliptic. In the first case the genus of $Y$ is $(\operatorname{deg}(D)+2) / 2=3$ giving a contradiction. In the second case, $Y$ is a hyperelliptic curve dominating $X$, therefore $X$ is also hyperelliptic by 38 , Proposition 1], and we also have a contradiction.

The following lemma appears to be implicitly assumed in Manoharmayum's argument [51, page 710], and is perhaps a known fact about the Klein quartic, although we have been unable to find it in the literature, and therefore give a proof.

Lemma 7.3. The only real twist of the Klein quartic is itself.

Proof. Write $X=X(7)$ for the Klein quartic (4). Its real twists are classified by $\mathrm{H}^{1}(\operatorname{Gal}(\mathbb{C} / \mathbb{R}), \operatorname{Aut}(X))$, where $\operatorname{Aut}(X)$ is the automorphism group of $X / \mathbb{C}$. The group $\operatorname{Aut}(X)$ is the unique simple group of order 168. Generators for $\operatorname{Aut}(X)$ as a subgroup of $\mathrm{PGL}_{3}(\mathbb{C})$ were given by Klein, and are reproduced by Elkies in [28, page 54]. We used these to explicitly write down the semidirect product

$$
\operatorname{Gal}(\mathbb{C} / \mathbb{R}) \ltimes \operatorname{Aut}(X) .
$$

There are 28 complements of $\operatorname{Aut}(X)$ in this semidirect product and we verified that they form a single conjugacy class, therefore $\mathrm{H}^{1}(\operatorname{Gal}(\mathbb{C} / \mathbb{R}), \operatorname{Aut}(X))=0$. 


\section{On Modularity over Totally Real Fields: Proof of Theorem 5}

Let $K$ be a totally real field and $E$ an elliptic curve over $K$. By Theorems 3 and 4 we know that $E$ is modular except possibly if $\bar{\rho}_{E, p}\left(G_{K\left(\zeta_{p}\right)}\right)$ is simultaneously absolutely reducible for $p=3,5,7$. It follows from Proposition 4.1 that such $E$ gives rise to a $K$-point on one of the 27 modular curves in (11). These all have genera $>1$, and so by Faltings' Theorem [30] at most finitely many $K$-points. Theorem 5 follows immediately.

Remark. By studying the gonalities of various modular curves, the second-named author [49] proves much stronger finiteness statements than Theorem [5.

\section{Part 4. A More Precise Study of Images}

\section{The Image $\bar{\rho}_{E, p}\left(G_{K\left(\zeta_{p}\right)}\right)$ Again}

The following proposition gives a refinement of Proposition 4.1 for $p=3,5,7$. Part (a) of the proposition (corresponding to $p=3$ ) is in fact proved by Rubin in 63, Proposition 6]. The group denoted by $H$ in part (b) appears (implicitly) in a similar setting in [17, Proof of Lemma 7.2.3].

Proposition 9.1. Let $K$ be a totally real number field, and let $E$ be an elliptic curve over $K$. Let $p$ be a rational prime and suppose

$$
K \cap \mathbb{Q}\left(\zeta_{p}\right)=\mathbb{Q} .
$$

Write $\bar{\rho}=\bar{\rho}_{E, p}$. Suppose $\bar{\rho}$ is irreducible but $\bar{\rho}\left(G_{K\left(\zeta_{p}\right)}\right)$ is absolutely reducible.

(a) If $p=3$ then $\bar{\rho}\left(G_{K}\right)$ is conjugate to $C_{\mathrm{s}}^{+}(3)$ (the normalizer of a split Cartan subgroup of $\mathrm{GL}_{2}\left(\mathbb{F}_{3}\right)$ ); this has order 8 , and its image in $\mathrm{PGL}_{2}\left(\mathbb{F}_{3}\right)$ is isomorphic to $(\mathbb{Z} / 2 \mathbb{Z})^{2}$.

(b) If $p=5$ then $\bar{\rho}\left(G_{K}\right)$ is conjugate in $\mathrm{GL}_{2}\left(\mathbb{F}_{5}\right)$ to the subgroup

$$
H=\left\langle\left(\begin{array}{ll}
0 & 1 \\
2 & 0
\end{array}\right),\left(\begin{array}{ll}
1 & 0 \\
0 & 4
\end{array}\right)\right\rangle
$$

of order 16. The subgroup $H$ is contained as a subgroup of index 2 in the normalizer of a split Cartan subgroup, and as a subgroup of index 3 in the normalizer of a non-split Cartan subgroup. The image of $H$ in $\mathrm{PGL}_{2}\left(\mathbb{F}_{5}\right)$ is isomorphic to $(\mathbb{Z} / 2 \mathbb{Z})^{2}$.

(c) If $p=7$ then $\bar{\rho}\left(G_{K}\right)$ is conjugate in $\mathrm{GL}_{2}\left(\mathbb{F}_{7}\right)$ to a subgroup of either $H_{1}$ or $\mathrm{H}_{2}$ where

$$
H_{1}=\left\langle\left(\begin{array}{ll}
3 & 0 \\
0 & 5
\end{array}\right),\left(\begin{array}{ll}
0 & 2 \\
2 & 0
\end{array}\right)\right\rangle, \quad H_{2}=\left\langle\left(\begin{array}{ll}
0 & 5 \\
3 & 0
\end{array}\right),\left(\begin{array}{ll}
5 & 0 \\
3 & 2
\end{array}\right)\right\rangle .
$$

The group $H_{1}$ has order 36 and is contained as a subgroup of index 2 in the normalizer of a split Cartan subgroup. The group $\mathrm{H}_{2}$ has order 48 and is contained as a subgroup of index 2 in the normalizer of a non-split Cartan subgroup. The images of $H_{1}$ and $H_{2}$ in $\mathrm{PGL}_{2}\left(\mathbb{F}_{7}\right)$ are respectively isomorphic to $D_{3} \cong S_{3}$ and $D_{4}$.

Proof. Write $G=\bar{\rho}\left(G_{K}\right)$. We observe that $G$ satisfies the following:

(i) $G$ is irreducible, and $G \cap \mathrm{SL}_{2}\left(\mathbb{F}_{p}\right)$ is absolutely reducible; 
(ii) $G$ odd (in other words, there is some element $c \in G$ with eigenvalues 1 and $-1)$

(iii) $\operatorname{det}(G)=\mathbb{F}_{p}^{*}$.

Indeed, (i) follows by assumption and Proposition 4.1, (ii) is a consequence of $K$ having a real embedding, and (iii) follows from $K \cap \mathbb{Q}\left(\zeta_{p}\right)=\mathbb{Q}$. One proof of the proposition is to enumerate all subgroups $G$ of $\mathrm{GL}_{2}\left(\mathbb{F}_{p}\right)$ with $p=3,5,7$, and check that those satisfying (i), (ii), (iii), also satisfy (a), (b), (c). We can simplify the enumeration by using Lemma 4.2 and its proof, from which we know that $G \cap \mathrm{GL}_{2}^{+}\left(\mathbb{F}_{p}\right)$ is contained in a Cartan subgroup $C_{*}(p)$ and $G$ is contained in its normalizer $C_{*}^{+}(p)$. If $p=5$ then the element $c \in G$ that represents complex conjugation belongs to $\mathrm{GL}_{2}^{+}\left(\mathbb{F}_{p}\right)$ and therefore to $G \cap \mathrm{GL}_{2}^{+}\left(\mathbb{F}_{p}\right)$. Now an element of $C_{\mathrm{ns}}(5)$ has eigenvalues $\alpha, \alpha^{p}$ for some $\alpha \in \mathbb{F}_{25}^{*}$. This shows that the non-split Cartan case does not arise for $p=5$.

Remark. It follows from the proof that $\bar{\rho}\left(G_{L}\right)$ is contained in a Cartan subgroup, where $L=K\left(\sqrt{(-1)^{(p-1) / 2} p}\right)$.

\section{Modular Curves Again}

Let $H_{1}, H_{2}$ be the two subgroups of $\mathrm{GL}_{2}\left(\mathbb{F}_{7}\right)$ specified in part (c) of Proposition 9.1. We shall denote the corresponding modular curves $X\left(H_{1}\right)$ and $X\left(H_{2}\right)$ by $X(\mathrm{~d} 7)$ and $X(\mathrm{e} 7)$ respectively. We extend our earlier conventions regarding the naming of modular curves in an obvious way. Thus, for a number field $K$, noncuspidal $K$-points on $X(\mathrm{~s} 3, \mathrm{~b} 5, \mathrm{~d} 7)$ give elliptic curves $E / K$ such that the image of $\bar{\rho}_{E, 3}$ is contained in a conjugate of the normalizer of a split Cartan subgroup of $\mathrm{GL}_{2}\left(\mathbb{F}_{3}\right)$, the image of $\bar{\rho}_{E, 5}$ is contained in a conjugate of the Borel subgroup of $\mathrm{GL}_{2}\left(\mathbb{F}_{5}\right)$, and the image of $\bar{\rho}_{E, 7}$ is contained in a conjugate of the group $H_{1}$.

Corollary 10.1. Let $K$ be a real quadratic field, and let $E$ be an elliptic curve defined over $K$.

(a) If $\bar{\rho}_{E, 3}\left(G_{K\left(\zeta_{3}\right)}\right)$ is absolutely reducible then $E$ gives rise to a non-cuspidal $K$-point on either $X(\mathrm{~b} 3)$ or $X(\mathrm{~s} 3)$.

(b) If $K \neq \mathbb{Q}(\sqrt{5})$ and $\bar{\rho}_{E, 5}\left(G_{K\left(\zeta_{5}\right)}\right)$ is absolutely reducible then $E$ gives rise to a non-cuspidal $K$-point on either $X(\mathrm{~b} 5)$ or $X(\mathrm{~s} 5)$.

(c) If $\bar{\rho}_{E, 7}\left(G_{K\left(\zeta_{7}\right)}\right)$ is absolutely reducible then $E$ gives rise to a non-cuspidal $K$-point on either $X(\mathrm{~b} 7)$ or $X(\mathrm{~d} 7)$ or $X(\mathrm{e} 7)$.

Proof. The field $K$ is real quadratic, so for $p=3$, 7, we have $K \cap \mathbb{Q}\left(\zeta_{p}\right)=\mathbb{Q}$. For $p=5$ this holds once we have imposed the condition $K \neq \mathbb{Q}(\sqrt{5})$. The corollary follows from Proposition 9.1.

\section{Remarks.}

(i) In (b) we could have replaced $X(\mathrm{~s} 5)$ with the curve $X(H)$, where $H$ is as given in the statement of Proposition 9.1. In fact $X(H)$ is a double cover of $X(\mathrm{~s} 5)$, so this would give a more precise statement. However, for our later purpose of proving modularity of elliptic curves over real quadratic fields, we have found it sufficient and simpler to work with $X$ (s5).

(ii) Parts (a), (b) improve over Proposition 4.1 by eliminating $X$ (ns3) and $X(n s 5)$. This substantially reduces the amount of work we have to do later in enumerating quadratic points on modular curves. 
(iii) In (b) we really need the condition $K \neq \mathbb{Q}(\sqrt{5})$. To see this let $K=\mathbb{Q}(\sqrt{5})$. Let $G=\bar{\rho}\left(G_{K}\right)$ where $\bar{\rho}=\bar{\rho}_{E, 5}$ and $E / K$ is an elliptic curve. Then

$$
\operatorname{det}(G)=\chi_{5}\left(\operatorname{Gal}\left(\mathbb{Q}\left(\zeta_{5}\right) / \mathbb{Q}(\sqrt{5})\right)\right)=\{\overline{1}, \overline{4}\} \subset \mathbb{F}_{5}^{*} .
$$

Now, in the proof of Proposition 9.1, if we replace the condition $\operatorname{det}(G)=\mathbb{F}_{5}^{*}$ with $\operatorname{det}(G)=\{\overline{1}, \overline{4}\}$ we obtain three subgroups of $\mathrm{GL}_{2}\left(\mathbb{F}_{5}\right)$ of orders 6 , 8 and 12. The subgroup of order 8 is contained in the normalizer of a split Cartan subgroup, but not in the normalizer of any non-split Cartan subgroup. The subgroups of orders 6 and 12 are contained in the normalizer of a non-split Cartan subgroup but not in the normalizer of any split Cartan subgroup.

\section{TheOrem 1 follows From Lemmas 1.1 and 1.2}

Most of the rest of this paper is devoted to the proof of Lemma 1.2, which states that non-cuspidal real quadratic points on the modular curves in (2) and (3) are modular. Let $X$ be any of the modular curves in (2), (3). Recall that $X$ is a moduli space of elliptic curves with level structures. A non-cuspidal point on $X$ gives rise to a well-defined $j$-invariant, and when $j \neq 0,1728$ this determines the underlying elliptic curve up to quadratic twists. When we say that a point on $X$ is modular, we mean that one (and hence any) elliptic curve supporting it is modular. Lemma 1.2 is in fact a summary of results established separately in the following lemmas: Lemma 12.1. Lemma 13.1. Lemma 14.1 and Lemma 17.1.

Lemma 1.1 asserts modularity of elliptic curves over $\mathbb{Q}(\sqrt{5})$. Its proof is relegated to Section 18. We now explain how Lemmas 1.1 and 1.2 are enough to imply the modularity of all elliptic curves over real quadratic fields.

Proof of Theorem 1, Let $E$ be an elliptic curve over a real quadratic field $K$. We would like to show that $E$ is modular. By Lemma 1.1 we may suppose that $K \neq \mathbb{Q}(\sqrt{5})$. We know from Theorems 3 and 4 that if $\bar{\rho}_{E, p}\left(G_{K\left(\zeta_{p}\right)}\right)$ is absolutely irreducible with $p=3$ or 5 or 7 then $E$ is modular. Thus we may restrict our attention to elliptic curves $E$ such that $\bar{\rho}_{E, p}\left(G_{K\left(\zeta_{p}\right)}\right)$ is simultaneously absolutely reducible for $p=3,5$ and 7 . Now appealing to Corollary 10.1 we see that $E$ gives rise to a $K$-point on one of the twelve modular curves

$$
\begin{array}{rrr}
X(\mathrm{~b} 3, \mathrm{~b} 5, \mathrm{~b} 7), & X(\mathrm{~b} 3, \mathrm{~b} 5, \mathrm{~d} 7), & X(\mathrm{~b} 3, \mathrm{~b} 5, \mathrm{e} 7), \\
X(\mathrm{~b} 3, \mathrm{~s} 5, \mathrm{~b} 7), & X(\mathrm{~b} 3, \mathrm{~s} 5, \mathrm{~d} 7), & X(\mathrm{~b} 3, \mathrm{~s} 5, \mathrm{e} 7), \\
X(\mathrm{~s} 3, \mathrm{~b} 5, \mathrm{~b} 7), & X(\mathrm{~s} 3, \mathrm{~b} 5, \mathrm{~d} 7), & X(\mathrm{~s} 3, \mathrm{~b} 5, \mathrm{e} 7), \\
X(\mathrm{~s} 3, \mathrm{~s} 5, \mathrm{~b} 7), & X(\mathrm{~s} 3, \mathrm{~s} 5, \mathrm{~d} 7), & X(\mathrm{~s} 3, \mathrm{~s} 5, \mathrm{e} 7) .
\end{array}
$$

It is clear that every one of these twelve covers one of the seven modular curves in Lemma 1.2, which completes the proof.

The proof of Lemma 1.2 will involve the determination of all non-cuspidal real quadratic points on some of the seven modular curves. If these points correspond to $\mathrm{CM}$ elliptic curves or $\mathbb{Q}$-curves then they are modular (see the remark at the beginning of Section 12 for more on the latter). The following lemma will sometimes be useful in proving the modularity of the remaining points. 
Lemma 11.1. Let $E$ be an elliptic curve over a totally real field $K$, with $j$-invariant $j$. Let $p=3$ or 5 or 7 , and suppose that the $p$-th division polynomial of $E$ is irreducible. Let $\mathfrak{q}$ be a prime of $K$ satisfying $v_{\mathfrak{q}}(j)<0$ and $p+v_{\mathfrak{q}}(j)$. Then $E$ is modular.

Proof. We shall show that $\bar{\rho}_{E, p}\left(G_{K}\right)$ contains $\mathrm{SL}_{2}\left(\mathbb{F}_{p}\right)$, which is enough to imply modularity by Theorems 3 and 4 .

By the theory of the Tate curve [71, Proposition 6.1], the two conditions $v_{\mathfrak{q}}(j)<0$ and $p+v_{\mathfrak{q}}(j)$ together imply that $p$ divides the order of $\bar{\rho}_{E, p}\left(G_{K}\right)$. It follows from the classification of maximal subgroups of $\mathrm{GL}_{2}\left(\mathbb{F}_{p}\right.$ ) (see for example 78, Lemma 2]) that $\bar{\rho}_{E, p}\left(G_{K}\right)$ is either contained in a Borel subgroup or contains $\mathrm{SL}_{2}\left(\mathbb{F}_{p}\right)$. Since the $p$-th division polynomial is irreducible, $\bar{\rho}_{E, p}\left(G_{K}\right)$ is not contained in a Borel subgroup.

\section{Remarks.}

(I) See [49] for an alternative study of modular curves and quadratic points on them related to elliptic curves with small residual image at 3,5 , and 7 .

(II) It is natural to ask whether the quadratic points on the seven modular curves in Lemma 1.2 can determined by the methods of Mazur [54], Kamienny [42] and Merel [55]. Let $X$ be any of these modular curves. To apply such methods to our problem we appear to require:

(a) an abelian variety $\mathcal{A} / \mathbb{Q}$ having Mordell-Weil rank 0 , that is a quotient of the Jacobian $J$ of $X$;

(b) a prime $p$ and a morphism $X^{(2)} \rightarrow \mathcal{A}$ (defined over $\mathbb{Q}$ ) that is injective on some small $p$-adic neighbourhood $U \subset X^{(2)}\left(\mathbb{Q}_{p}\right)$ (where $X^{(2)}$ is the symmetric square of $X$ );

(c) some argument that shows that $X^{(2)}(\mathbb{Q}) \subset U$.

Usually $U$ is the $p$-adic residue class of $\{\infty, \infty\}$, and property (b) is a consequence of $X^{(2)} \rightarrow \mathcal{A}$ being a formal immersion at $\{\infty, \infty\}$ above $p$. For the modular curves that we consider, we have been unable to demonstrate (c) without actually determining the quadratic points 2. In fact, as we will see in due course, $X(\mathrm{~b} 5, \mathrm{~b} 7)$ and $X(\mathrm{~s} 3, \mathrm{~s} 5)$ have infinitely many quadratic points, so (b) and (c) cannot simultaneously hold. Likewise, $X(\mathrm{~b} 3, \mathrm{~s} 5)$ has many non-cuspidal quadratic points, suggesting that these methods are inapplicable.

\section{Part 5. Real Quadratic Points on Certain Modular Curves}

\section{Real Quadratic Points on $X(\mathrm{~b} 5, \mathrm{~b} 7)$}

In this section we parametrize the non-cuspidal real quadratic points on $X(\mathrm{~b} 5, \mathrm{~b} 7)$ (commonly known as $X_{0}(35)$ ), showing that they are all modular. In fact, we shall show that all except two correspond to $\mathbb{Q}$-curves. By a $\mathbb{Q}$-curve, we mean an elliptic curve defined over $\overline{\mathbb{Q}}$ that is isogenous to all its Galois conjugates. It is known that $\mathbb{Q}$-curves are modular. Indeed, Ribet [60, Corollary 6.2] had shown that modularity of $\mathbb{Q}$-curves is a consequence of Serre's modularity conjecture, which is now a theorem of Khare and Wintenberger [43, 44].

\footnotetext{
${ }^{2}$ Indeed, the absence of (c) is the reason why we do not yet have a parametrization of quadratic points on the family $X(\mathrm{~b} N)$, even though (a) and (b) are known [42] for prime $N>71$, where $\mathcal{A}$ is the Eisenstein quotient of $J_{0}(N)$, and $p$ is any prime $\neq 2,3,5$.
} 
Lemma 12.1. Let $X=X(\mathrm{~b} 5, \mathrm{~b} 7)$. The non-cuspidal real quadratic points on $X$ are modular.

Proof. The curve $X$ is hyperelliptic of genus 3. Galbraith [35, Section 4.4] derives the following model for $X$,

$$
X: y^{2}=\left(x^{2}+x-1\right)\left(x^{6}-5 x^{5}-9 x^{3}-5 x-1\right) .
$$

Write $J$ for $J_{0}(35)$ - the Jacobian of $X$. Using 2-descent [75] we find that $J(\mathbb{Q})$ has Mordell-Weil rank 0. As $J$ has good reduction at 3, the map $J(\mathbb{Q}) \rightarrow J\left(\mathbb{F}_{3}\right)$ is injective. The group generated by differences of the four obvious rational points $(0, \pm 1), \infty_{ \pm}$(where the latter are the points at infinity) surjects onto $J\left(\mathbb{F}_{3}\right) \cong$ $\mathbb{Z} / 24 \mathbb{Z} \times \mathbb{Z} / 2 \mathbb{Z}$, and is therefore equal to $J(\mathbb{Q})$. We find

$$
J(\mathbb{Q})=\frac{\mathbb{Z}}{24 \mathbb{Z}} \cdot\left[\infty_{-}-\infty_{+}\right]+\frac{\mathbb{Z}}{2 \mathbb{Z}} \cdot\left[3(0,-1)-3 \infty_{+}\right] .
$$

Let $P$ be a quadratic point of $X$, and let $P^{\sigma}$ denote its conjugate. Then $P+P^{\sigma}-$ $\infty_{+}-\infty_{-}$is a rational divisor of degree 0 and hence defines an element of $J(\mathbb{Q})$. It follows that $P+P^{\sigma}$ is linearly equivalent to $T+\infty_{+}+\infty_{-}$where $T$ is a divisor of degree 0 representing one of the 48 elements of $J(\mathbb{Q})$. As $X$ is hyperelliptic, for $[T] \neq 0$, the divisor $T+\infty_{+}+\infty_{-}$is linearly equivalent to at most one effective divisor of degree 2 . Enumerating these 47 possibilities for $[T] \neq 0$, we find that all the effective degree 2 divisors they yield are sums of two rational points, except for $\left(\frac{-1 \pm \sqrt{5}}{2}, 0\right)$. We would like to show that this pair of points is modular. By Ogg [59, Corollary 2], the hyperelliptic involution on $X=X(\mathrm{~b} 5, \mathrm{~b} 7)$ is the Fricke involution $w_{35}$. The points $\left(\frac{-1 \pm \sqrt{5}}{2}, 0\right)$ are fixed by $w_{35}$ and so correspond to CM elliptic curves, which are therefore modular.

We now turn to the case $[T]=0$, and so $P+P^{\sigma}$ is linearly equivalent to $\infty_{+}+\infty_{-}$. Thus $P=(x, \pm \sqrt{f(x)})$ with $x \in \mathbb{Q} \backslash\{0\}$, where $f$ is the polynomial on the right-hand side of (7). Suppose now that $P$ is a quadratic point of the form $(x, \sqrt{f(x)})$ with $x \in \mathbb{Q} \backslash\{0\}$. The Galois conjugate $P^{\sigma}$ of $P$ is related to $P$ by the hyperelliptic involution. Therefore the elliptic curve $E$ represented by $P \in X$ is related to its Galois conjugate $E^{\sigma}$ by a 35 -isogeny, and hence is a $\mathbb{Q}$-curve.

\section{Real Quadratic Points on $X(\mathrm{~b} 3, \mathrm{~s} 5)$}

Lemma 13.1. Let $X=X(\mathrm{~b} 3, \mathrm{~s} 5)$. All non-cuspidal real quadratic points on $X$ are modular.

Proof. The curve $X$ is studied briefly in [17, page 554-556] where it is shown that $X \cong X_{0}(75) / w_{25}$. That paper does not give a model for $X$ but mentions a computation by Elkies where he finds $X$ to have genus 3 and determines its rational points. It turns out there is precisely one non-cuspidal rational point and that this has $j$-invariant 0 .

In order to write down a model for $X$, and to compute the Mordell-Weil group of its Jacobian, we shall make use of Stein's Magma implementation of modular symbols algorithms [20], 74]. We find that an echelon basis for the +1-eigenspace 
of the Atkin-Lehner operator $w_{25}$ acting on $S_{2}\left(\Gamma_{0}(75)\right)$ is given by

$$
\begin{aligned}
& f_{1}=q-q^{4}-2 q^{5}+q^{6}+q^{9}+2 q^{10}-4 q^{11}+\cdots \\
& f_{2}=q^{2}-q^{4}+q^{6}-q^{7}-q^{8}-2 q^{11}+\cdots \\
& f_{3}=q^{3}+q^{4}+2 q^{5}-q^{6}+q^{7}-2 q^{8}-2 q^{10}+2 q^{11}+\cdots
\end{aligned}
$$

Thus $X$ has genus 3 ; a basis for the holomorphic differentials is given by $f_{i} d q / q$. Following Galbraith [35, Chapter 3], we compute the image of this curve under the canonical map, and find that it is a plane quartic curve. It follows that the canonical map is indeed an embedding of $X$ in $\mathbb{P}^{2}$. The equation given by the canonical embedding is

$$
\begin{aligned}
X: 3 x^{3} z-3 x^{2} y^{2}+5 x^{2} z^{2}-3 x y^{3}-19 x y^{2} z-x y z^{2} & \\
& +3 x z^{3}+2 y^{4}+7 y^{3} z-7 y^{2} z^{2}-3 y z^{3}=0 .
\end{aligned}
$$

We shall also need the map $j: X \rightarrow X(1)$. The details of finding this map explicitly are similar to those in a recent paper of Banwait and Cremona [2, Section 5], and so we only give a sketch. The isomorphism $X_{0}(75) / w_{25} \rightarrow X$ pulled back to the upper half-plane $\mathfrak{H}$ is given by $\tau \mapsto 5 \tau$. Thus the $q$-expansion of $j$ on $X$ is given by

$$
j\left(q^{5}\right)=\frac{1}{q^{5}}+744+196884 q^{5}+21493760 q^{10}+864299970 q^{15}+20245856256 q^{20}+\cdots .
$$

We can express $j=F(x, y, z) / G(x, y, z)$ where $F, G$ are homogeneous forms of the same degree with coefficients in $\mathbb{Z}$. We seek such $F, G$ so that the identity of modular forms

$$
F\left(f_{1}, f_{2}, f_{3}\right)=j\left(q^{5}\right) G\left(f_{1}, f_{2}, f_{3}\right)
$$

holds. To find suitable $F, G$, we run through the degrees $d=1,2, \ldots$ and express $F$, $G$ as linear combinations of the monomials of degree $d$ with unknown coefficients. By comparing the coefficients of the $q$-expansions in (91) we obtain simultaneous equations for the unknown coefficients of $F, G$. Solving these we obtain the first non-zero $F, G$ with $d=15$. For this particular $F, G$ we checked the equality of coefficients on both sides of (9) beyond the Sturm bound [74, Theorem 9.18]. This is sufficient to show that $j=F(x, y, z) / G(x, y, z)$. The expressions for $F$ and $G$ are complicated and can be found in the accompanying Magma script (see page 5).

Write $J$ for the Jacobian of $X$. Computing the eigenbasis for the space spanned by $f_{1}, f_{2}, f_{3}$ yields three rational eigenforms corresponding to the elliptic curves $E_{1}, E_{2}, E_{3}$ with Cremona labels $15 A 1,75 B 1,75 C 1$. It follows that $J$ is isogenous to $E_{1} \times E_{2} \times E_{3}$.

We use this to compute the rank of $J(\mathbb{Q})$ and of $J(\mathbb{Q}(\sqrt{5})$ ) (we will see in due course why the latter rank is convenient to have). The three curves $E_{i}$ have rank 0 over $\mathbb{Q}$ and so do their quadratic twists by by 5 . Thus $E_{i}$ have rank 0 over $\mathbb{Q}$ and over $\mathbb{Q}(\sqrt{5})$. It follows that $J$ has rank 0 over those two fields. Thus $J(\mathbb{Q})$ is equal to its torsion subgroup. Let $L=\mathbb{Q}(\sqrt{5})$. We shall denote the torsion subgroups of $J(\mathbb{Q})$ and of $J(L)$ by $J(\mathbb{Q})_{\text {tors }}$ and $J(L)_{\text {tors }}$. To compute $J(\mathbb{Q})_{\text {tors }}$ we shall in fact compute $J(L)_{\text {tors }}$ and recover $J(\mathbb{Q})_{\text {tors }}$ using the inclusion $J(\mathbb{Q})_{\text {tors }} \subseteq J(L)_{\text {tors }}$. A 
short search for points on $X$ defined over $L$ reveals the following points:

$$
\begin{gathered}
P_{0}=(0: 0: 1), \quad P_{1}=(1: 0: 0), \quad P_{2}=(1:-1: 2), \\
P_{3}=\left(-2: \frac{-1+\sqrt{5}}{2}: 1\right), \quad P_{4}=\left(\frac{1-\sqrt{5}}{2}:-3+\sqrt{5}: 1\right), \quad P_{5}=\left(\frac{1+\sqrt{5}}{2}: 2+\sqrt{5}: 1\right) .
\end{gathered}
$$

As $J(L)$ is torsion, we see that $\left[P_{i}-P_{0}\right]$ belong to $J(L)_{\text {tors }}$. Write $H$ for the subgroup of $J(L)_{\text {tors }}$ generated by the differences $\left[P_{i}-P_{0}\right],\left[P_{i}^{\sigma}-P_{0}\right]$ where $\sigma$ denotes conjugation in $L$. Write $\mathcal{O}_{L}$ for the ring of integers of $L$ and let $\mathfrak{p}=$ $19 \mathcal{O}_{L}+(9+\sqrt{5}) \mathcal{O}_{L}$. Then $\mathfrak{p}$ is a prime ideal of norm 19. Thus we have a natural injection

induced by $\mathfrak{p}$. We find that

$$
\pi_{\mathfrak{p}}: J(L)_{\text {tors }} \hookrightarrow J\left(\mathbb{F}_{19}\right)
$$

$J\left(\mathbb{F}_{19}\right) \cong \mathbb{Z} / 2 \mathbb{Z} \times \mathbb{Z} / 2 \mathbb{Z} \times \mathbb{Z} / 8 \mathbb{Z} \times \mathbb{Z} / 200 \mathbb{Z}, \quad \pi_{\mathfrak{p}}(H) \cong \mathbb{Z} / 2 \mathbb{Z} \times \mathbb{Z} / 2 \mathbb{Z} \times \mathbb{Z} / 8 \mathbb{Z} \times \mathbb{Z} / 40 \mathbb{Z}$.

It follows that either $J(L)_{\text {tors }}=H$ or $H$ has index 5 in $J(L)_{\text {tors. }}$ In the latter case, the order of $J(L)_{\text {tors }}$ is divisible by 25 .

Let $\mathfrak{q}=7 \mathcal{O}_{L}$. This is a prime ideal of norm 49 , and so we have another injection

$$
\pi_{\mathfrak{q}}: J(L)_{\text {tors }} \hookrightarrow J\left(\mathbb{F}_{49}\right) \cong(\mathbb{Z} / 8 \mathbb{Z})^{3} \times \mathbb{Z} / 440 \mathbb{Z} \text {. }
$$

As the order of $J\left(\mathbb{F}_{49}\right)$ is not divisible by 25 , the order of $J(L)_{\text {tors }}$ is not divisible by 25 . It follows that $J(L)_{\text {tors }}=H$. Taking $\operatorname{Gal}(L / \mathbb{Q})$-invariants, we find that

$$
J(\mathbb{Q})=J(\mathbb{Q})_{\text {tors }}=(\mathbb{Z} / 40 \mathbb{Z}) \cdot\left[P_{2}-P_{0}\right]+(\mathbb{Z} / 2 \mathbb{Z}) \cdot\left[P_{3}+P_{3}^{\sigma}+3 P_{0}-5 P_{2}\right] .
$$

We now enumerate the real quadratic points of $X$. As $X$ is a plane quartic (and therefore not hyperelliptic), the map

$$
X^{(2)}(\mathbb{Q}) \rightarrow J(\mathbb{Q}), \quad D \mapsto\left[D-2 P_{0}\right]
$$

is injective. Note that any element $D \in X^{(2)}(\mathbb{Q})$ is linearly equivalent to $D^{\prime}+2 P_{0}$, where $\left[D^{\prime}\right] \in J(\mathbb{Q})$. For each of the 80 elements $\left[D^{\prime}\right]$ of $J(\mathbb{Q})$ we computed the Riemann-Roch space of $D^{\prime}+2 P_{0}$ and checked if this divisor is linearly equivalent to a positive divisor of degree 2 (in which case the positive divisor is unique). This allowed us to enumerate $X^{(2)}(\mathbb{Q})$ and hence all the rational and quadratic points on $X$. It turns out that

$$
X(\mathbb{Q})=\left\{P_{0}, P_{1}, P_{2}\right\} .
$$

The points $P_{0}$ and $P_{1}$ are cusps; the point $P_{2}$ has $j$-invariant 0 . This is consistent with Elkies' computation mentioned in [17, page 555]. The complex quadratic points are (up to conjugation)

$$
(-5+\sqrt{-11}: 0: 6), \quad(-6+2 \sqrt{-15}: 1+\sqrt{-15}: 8),
$$

with $j$-invariants -32768 and 0 respectively. Table 1 gives the real quadratic points (up to conjugation), and their $j$-invariants; the ones with $j$-invariant $=\infty$ are of course cusps.

From the table we see that there are 12 non-cuspidal real quadratic points (up to conjugation). Of these, seven correspond to elliptic curves with complex multiplication and are therefore modular. For the remaining five modularity follows from Lemma 11.1 with $p=7$ and $\mathfrak{q}$ some prime above 2 . 


\begin{tabular}{|c|c|c|}
\hline point & $j$-invariant & $\begin{array}{c}\text { discriminant of quadratic } \\
\text { order of CM }\end{array}$ \\
\hline \hline$(-2+\sqrt{2}: \sqrt{2}: 2)$ & $2417472-1707264 \sqrt{2}$ & -24 \\
\hline$(-1:-2+\sqrt{3}: 1)$ & 1728 & -4 \\
\hline$(-2:-1+\sqrt{3}: 2)$ & $76771008+44330496 \sqrt{3}$ & -36 \\
\hline$(1+\sqrt{5}:-6-2 \sqrt{5}: 2)$ & 0 & -3 \\
\hline$(1+\sqrt{5}: 4+2 \sqrt{5}: 2)$ & $\infty$ & not applicable \\
\hline$(-4:-1+\sqrt{5}: 2)$ & $-2770550784-671956992 \sqrt{17}$ & not applicable \\
\hline$(-10+2 \sqrt{17}: 3-\sqrt{17}: 4)$ & -32768 & -51 \\
\hline$(-3+\sqrt{33}: 6: 0)$ & $-18808030478336+3274057859072 \sqrt{33}$ & -11 \\
\hline$(-9+\sqrt{33}:-9+\sqrt{33}: 8)$ & $(-251512901-39335769 \sqrt{41}) / 64$ & -99 \\
\hline$(-8:-11+\sqrt{41}: 4)$ & $(3494273-283495 \sqrt{145}) / 64$ & non-CM \\
\hline$(-5+\sqrt{41}:-2+2 \sqrt{41}: 8)$ & $(839560577-84563815 \sqrt{145}) / 65536$ & non-CM \\
\hline$(-23+\sqrt{145}:-12: 24)$ & 05536 & non-CM \\
\hline$(11+\sqrt{145}:-11-\sqrt{145}: 2)$ & non-CM \\
\hline$(-91+\sqrt{185}: 6-2 \sqrt{185}: 88)$ & $(572227476834375-42070984346475 \sqrt{185}) / 65536$ & non-CM \\
\hline
\end{tabular}

TABLE 1 . The table gives the real quadratic points on the curve $X(\mathrm{~b} 3, \mathrm{~s} 5)$ given by the model in (8). It also gives the $j$-invariants of these points, and, where applicable, the discriminant of the order of complex multiplication of the corresponding elliptic curve. 


\section{Real Quadratic Points on $X(\mathrm{~s} 3, \mathrm{~s} 5)$}

Lemma 14.1. Non-cuspidal real quadratic points on $X(\mathrm{~s} 3, \mathrm{~s} 5)$ are modular.

Proof. Let $X=X(\mathrm{~s} 3, \mathrm{~s} 5)$. As in the proof of Lemma13.1, we use the canonical map to write down a model for $X \cong X_{0}(225) /\left\langle w_{9}, w_{25}\right\rangle$. The subspace of $S_{2}\left(\Gamma_{0}(225)\right)$ invariant under $w_{9}$ and $w_{25}$ has dimension 4 and echelon basis

$$
\begin{aligned}
& f_{1}=q-4 q^{5}-4 q^{6}+5 q^{7}-4 q^{9}+4 q^{10}-8 q^{11}+\cdots \\
& f_{2}=q^{2}-2 q^{5}-4 q^{6}+4 q^{7}-q^{8}-2 q^{9}+2 q^{10}-6 q^{11}+\cdots, \\
& f_{3}=q^{3}-2 q^{5}-2 q^{6}+2 q^{7}+q^{8}-q^{9}+2 q^{10}-3 q^{11}+\cdots \\
& f_{4}=q^{4}-2 q^{5}-2 q^{6}+5 q^{7}-2 q^{9}+2 q^{10}-4 q^{11}+\cdots
\end{aligned}
$$

Thus $X$ has genus 4 . Instead of using the basis $f_{1}, \ldots, f_{4}$, we shall use the following basis:

$$
g_{1}=-f_{1}+2 f_{4}, \quad g_{2}=f_{1}-f_{2}+2 f_{3}-f_{4}, \quad g_{3}=f_{1}+f_{2}-2 f_{3}-f_{4}, \quad g_{4}=f_{1}-f_{3} .
$$

The motivation behind this change of basis is that the automorphisms of the model for $X$ given by the canonical map induced by $g_{1}, \ldots, g_{4}$ are easier to describe as we will soon see. The canonical map $X \rightarrow \mathbb{P}^{3}$ induced by $g_{1}, \ldots, g_{4}$ is an embedding and yields the following model:

$$
X:\left\{\begin{array}{l}
3 x_{1}^{2}-x_{2}^{2}-2 x_{3}^{2}+2 x_{3} x_{4}-3 x_{4}^{2}=0 \\
x_{2}^{2} x_{3}-x_{3}^{3}+4 x_{3}^{2} x_{4}-12 x_{3} x_{4}^{2}+12 x_{4}^{3}=0 .
\end{array}\right.
$$

Note that $X$ has the following pair of involutions,

$$
\alpha:\left(x_{1}, x_{2}, x_{3}, x_{4}\right) \mapsto\left(-x_{1}, x_{2}, x_{3}, x_{4}\right), \quad \beta:\left(x_{1}, x_{2}, x_{3}, x_{4}\right) \mapsto\left(x_{1},-x_{2}, x_{3}, x_{4}\right) .
$$

Write $\mathcal{A}:=\{1, \alpha, \beta, \alpha \beta\}$. (It is possible to show that $\mathcal{A}$ is the automorphism group of $X$, but we shall not need this.) Write $\mathbb{K}$ for the function field of $X$. The subfield fixed by $\mathcal{A}$ is $\mathbb{Q}(w)$ where $w=x_{3} / x_{4}$. Since $\mathcal{A} \cong \mathbb{Z} / 2 \mathbb{Z} \times \mathbb{Z} / 2 \mathbb{Z}$, it follows that $\mathbb{K}=\mathbb{Q}(w)\left(\sqrt{h_{1}}, \sqrt{h_{2}}\right)$ for some $h_{1}, h_{2} \in \mathbb{Q}(w)$. By inspection, we see that $X$ has the following more convenient model:

$$
X: \begin{cases}u^{2} & =w(w-1)\left(w^{2}-w+4\right) \\ v^{2} & =w\left(w^{3}-4 w^{2}+12 w-12\right)\end{cases}
$$

where

$$
u=\frac{x_{1} x_{3}}{x_{4}^{2}}, \quad v=\frac{x_{2} x_{3}}{x_{4}^{2}}, \quad w=\frac{x_{3}}{x_{4}} .
$$

We are interested in determining the real quadratic points of $X$, and for this we shall consider the following three obvious quotients

$$
\begin{aligned}
& D_{1}: u^{2}=w(w-1)\left(w^{2}-w+4\right), \\
& D_{2}: v^{2}=w\left(w^{3}-4 w^{2}+12 w-12\right), \\
& D_{3}: z^{2}=(w-1)\left(w^{2}-w+4\right)\left(w^{3}-4 w^{2}+12 w-12\right) .
\end{aligned}
$$

We shall write

$$
\begin{aligned}
& h_{1}=w(w-1)\left(w^{2}-w+4\right), \quad h_{2}=w\left(w^{3}-4 w^{2}+12 w-12\right), \\
& h_{3}=(w-1)\left(w^{2}-w+4\right)\left(w^{3}-4 w^{2}+12 w-12\right) .
\end{aligned}
$$

The quotient maps $\phi_{i}: X \rightarrow D_{i}$ are all of degree 2 , given by

$$
\phi_{1}(w, u, v)=(w, u), \quad \phi_{2}(w, u, v)=(w, v), \quad \phi_{3}(w, u, v)=(w, u v / w) .
$$


The curve $D_{2}$ is isomorphic to the Weierstrass elliptic curve with Cremona label $225 A 1$, with Mordell-Weil rank 1 over $\mathbb{Q}$ (and trivial torsion). It follows that $X$ has infinitely many quadratic points obtained by pulling back the rational points on $D_{2}$ via the degree 2 map $\phi_{2}$. We claim that all non-cuspidal real quadratic points on $X$ are obtained thus.

The curve $D_{3}$ has genus 2. Write $J$ for its Jacobian. Using 2-descent we find that $J(\mathbb{Q})$ has Mordell-Weil rank 0, and by a similar calculation to those in the previous sections we find that

$$
J(\mathbb{Q})=\frac{\mathbb{Z}}{10 \mathbb{Z}} \cdot\left[\left(\frac{1+\sqrt{5}}{2}, \frac{-15+5 \sqrt{5}}{2}\right)+\left(\frac{1-\sqrt{5}}{2}, \frac{-15-5 \sqrt{5}}{2}\right)-\infty_{+}-\infty_{-}\right]
$$

Here $\infty_{+}, \infty_{-}$are the two points at infinity on $D_{3}$. As in the proof of Lemma 12.1 we can determine all rational and quadratic points on $D_{3}$. In particular,

$$
D_{3}(\mathbb{Q})=\left\{(1,0), \infty_{+}, \infty_{-}\right\}
$$

The real quadratic points $(w, z)$ on $D_{3}$ are, up to Galois conjugation and the action of the hyperelliptic involution, equal to $((1+\sqrt{5}) / 2,(-15+5 \sqrt{5}) / 2)$, or satisfy $w \in \mathbb{Q}$ and $z= \pm \sqrt{h_{3}(w)} \notin \mathbb{Q}$.

Clearly a real quadratic point on $X$ maps to either a rational or a real quadratic point on $D_{3}$. We deduce that the only real quadratic points on $X$ satisfy

(a) either $(w, u, v)=((1-\sqrt{5}) / 2,(-5-\sqrt{5}) / 2, \sqrt{5})$ or its conjugates under the action of Galois and the automorphisms $u \mapsto-u, v \mapsto-v$;

(b) or $w \in \mathbb{Q}$ and $u v / w= \pm \sqrt{h_{3}(w)} \notin \mathbb{Q}$,

The points in (a) satisfy $w^{2}-w-1=0$, and are cusps as can be deduced from the expression for $j$-map on $X$ given below in (11).

We are interested in proving the modularity of the non-cuspidal real points $P=$ $(w, u, v)$ satisfying (b). Write $d=h_{3}(w)$. Let $K=\mathbb{Q}(\sqrt{d})$, which is the field of the definition of the point $P$. If $u$ is rational, then $\phi_{1}(P)=(w, u) \in D_{1}(\mathbb{Q})$. However, $D_{1}$ is isomorphic to the Weierstrass elliptic curve with Cremona label 15A8, with rank 0 and precisely four rational points. On the model $D_{1}$ the four points are the two points at infinity, and the points $(0,0)$ and $(1,0)$. None of these are images of real quadratic points on $X$. We can conclude that $u= \pm \sqrt{h_{1}(w)}$ is not rational. As $h_{1}(w) \in \mathbb{Q}$ and $u \in K=\mathbb{Q}(\sqrt{d})$ we have $h_{1}(w)=a^{2} d$ for some non-zero $a \in \mathbb{Q}$. Thus

$$
h_{2}(w)=\frac{w^{2} h_{3}(w)}{h_{1}(w)}=\frac{w^{2} d}{a^{2} d}=\frac{w^{2}}{a^{2}} .
$$

Therefore $v \in \mathbb{Q}$. Hence, if $P=(w, u, v)$ is a non-cuspidal real quadratic point on $X$ then $\phi_{2}(P)=(w, v) \in D_{2}(\mathbb{Q})$, proving our claim.

To prove that the non-cuspidal real quadratic points on $X$ are modular we need an expression for the $j$-map. The parametrization of $X$ via $g_{1}, \ldots, g_{4}$ allows us to write down the $j$-map as a rational function on $X$ using the same method as described in the proof of Lemma 13.1. On the model (10), the $j$-map is given by

$$
j=\frac{\left(R_{1}+R_{2} \cdot v\right)^{3}}{8 R_{3}^{15}}
$$


where

$$
\begin{aligned}
R_{1}= & w^{15}-10 w^{14}+45 w^{13}-115 w^{12}+155 w^{11}-54 w^{10}+185 w^{9}-2395 w^{8}+10465 w^{7} \\
& -27055 w^{6}+47072 w^{5}-56090 w^{4}+43405 w^{3}-19485 w^{2}+4020 w-132 \\
R_{2}= & w^{13}-8 w^{12}+25 w^{11}-35 w^{10}+5 w^{9}+94 w^{8}-467 w^{7}+1805 w^{6}-5175 w^{5} \\
& +9685 w^{4}-11216 w^{3}+7698 w^{2}-2715 w+315 ; \\
R_{3}= & w^{2}-w-1 .
\end{aligned}
$$

The expression for $j$ involves only $w$ and $v$. Thus the map $j: X \rightarrow X(1)$ factors via $\phi_{2}: X \rightarrow D_{2}$. If $P$ is a non-cuspidal real quadratic point on $X$ then $\phi_{2}(P) \in D_{2}(\mathbb{Q})$, and so $j(P) \in \mathbb{Q}$. The modularity of $P$ follows from the modularity of elliptic curves over the rationals 9 .

Remark. The reader might be surprised by the fact - crucial to our proof - that the $j$-map (11) on $X$ factors via the quotient $D_{2}$. Also surprising is the fact that the expression for $j$ is a perfect cube. To explain this, we start by the observation that the normalizer $C_{\mathrm{s}}^{+}(3)$ of a split Cartan subgroup in $\mathrm{GL}_{2}\left(\mathbb{F}_{3}\right)$ is conjugate to a subgroup of the normalizer $C_{\mathrm{ns}}^{+}(3)$ of a non-split Cartan subgroup. Thus the map $X(\mathrm{~s} 3) \rightarrow X(1)$ factors via $X(\mathrm{~ns} 3)$. Moreover, $C_{\mathrm{s}}^{+}(3) \cap \mathrm{SL}_{2}\left(\mathbb{F}_{3}\right)$ has index 2 in $C_{\mathrm{ns}}^{+}(3) \cap \mathrm{SL}_{2}\left(\mathbb{F}_{3}\right)$. It follows that the map $X(\mathrm{~s} 3) \rightarrow X(\mathrm{~ns} 3)$ has degree 2 . Thus the map $X \rightarrow X(1)$ factors via a degree 2 map $X \rightarrow X(\mathrm{~ns} 3$, s5). The curves $X$ (ns3) and $X(\mathrm{~s} 5)$ have genus 0 . In [14, Section 5.1], Chen derives the following expressions for the $j$-maps on $X(\mathrm{~ns} 3)$ and $X(\mathrm{~s} 5)$,

$$
j=n^{3}, \quad j=\frac{\left(s^{2}-5\right)^{3}\left(s^{2}+5 s+10\right)^{3}(s+5)^{3}}{\left(s^{2}+5 s+5\right)^{5}},
$$

where $n$ and $s$ are respectively Hauptmoduln on $X(\mathrm{~ns} 3)$ and $X(\mathrm{~s} 5)$. Since $X \rightarrow$ $X(1)$ factors via $X(\mathrm{~ns} 3)$, it is clear that $j$ must be a cube on $X$, and this provides a useful check on our computation of $j$. A (singular) model for $X(\mathrm{~ns} 3, \mathrm{~s} 5)$ is given by

$$
n^{3}=\frac{\left(s^{2}-5\right)^{3}\left(s^{2}+5 s+10\right)^{3}(s+5)^{3}}{\left(s^{2}+5 s+5\right)^{5}} .
$$

It is easy to see that this is birational to the elliptic curve

$$
E: s^{2}+5 s+5=m^{3} \text {. }
$$

The curve $E$ has Cremona label 225A1, and is in fact isomorphic to $D_{2}$. This explains why $X \rightarrow X(1)$ factors through $D_{2}$.

\section{Models for some Modular Curves of Genus 1}

We shall shortly study the quadratic points on the four modular curves in (3).

Lemma 15.1. The four modular curves in (3) respectively have genus 97, 153, 73 and 113 .

The lemma follows from a standard application of Riemann-Hurwitz applied to $X \rightarrow X(1)$, where $X$ runs through the four modular curves. For this, we need the ramification indices of points above 0,1728 and $\infty$, and we explain how to compute these in Section 16.1. We omit the details.

In view of the very great genera, it is unlikely that methods used to compute the quadratic points on the curves in (2) will succeed for the curves in (3). Indeed, 
it is entirely impractical to write down smooth projective models for any of these four curves. Instead, we shall view them as normalizations of the fibre products

$$
\begin{array}{ll}
X(\mathrm{~b} 3, \mathrm{~b} 5) \times_{X(1)} X(\mathrm{~d} 7), & X(\mathrm{~s} 3, \mathrm{~b} 5) \times_{X(1)} X(\mathrm{~d} 7), \\
X(\mathrm{~b} 3, \mathrm{~b} 5) \times_{X(1)} X(\mathrm{e} 7), & X(\mathrm{~s} 3, \mathrm{~b} 5) \times_{X(1)} X(\mathrm{e} 7) .
\end{array}
$$

We therefore write down models for

$$
X(\mathrm{~b} 3, \mathrm{~b} 5), \quad X(\mathrm{~s} 3, \mathrm{~b} 5), \quad X(\mathrm{~d} 7), \quad X(\mathrm{e} 7)
$$

and the corresponding $j$-maps to $X(1)$. We will also compute the Mordell-Weil groups of their Jacobians over $\mathbb{Q}$. We then use this information to study the quadratic points on the four curves in (3). The curves in (12) are all elliptic curves (genus 1 curves with $\mathbb{Q}$-points). Rather than identify elements of the Mordell-Weil groups with points on the curves (as is usual for elliptic curves), we shall view them as elements of $\mathrm{Pic}^{0}$; we have found this to be more convenient for studying quadratic points.

\subsection{The Modular Curves $X(\mathrm{~d} 7)$ and $X(\mathrm{e} 7)$.}

Lemma 15.2. A model for the curve $X(\mathrm{~d} 7)$ is given by

$$
X(\mathrm{~d} 7): y^{2}=-7\left(x^{4}-10 x^{3}+27 x^{2}-10 x-27\right) .
$$

The $j$-map $X(\mathrm{~d} 7) \rightarrow X(1)$ is given by

$$
j=\frac{x(x+1)^{3}\left(x^{2}-5 x+8\right)^{3}\left(x^{2}-5 x+1\right)^{3}\left(x^{4}-5 x^{3}+8 x^{2}-7 x+7\right)^{3}}{\left(x^{3}-4 x^{2}+3 x+1\right)^{7}} .
$$

The $\mathbb{Q}$-points on $X(\mathrm{~d} 7)$ are $(5 / 2, \pm 7 / 4)$. The Mordell-Weil group over $\mathbb{Q}$ is given by

$$
\frac{\mathbb{Z}}{2 \mathbb{Z}} \cdot[(5 / 2,-7 / 4)-(5 / 2,7 / 4)]
$$

A model for the curve $X(\mathrm{e} 7)$ is given by

$$
X(\mathrm{e} 7): y^{2}=7\left(16 x^{4}+68 x^{3}+111 x^{2}+62 x+11\right) .
$$

The $j$-map $X(\mathrm{e} 7) \rightarrow X(1)$ is given by

$$
j=\frac{(3 x+1)^{3}\left(4 x^{2}+5 x+2\right)^{3}\left(x^{2}+3 x+4\right)^{3}\left(x^{2}+10 x+4\right)^{3}}{\left(x^{3}+x^{2}-2 x-1\right)^{7}} .
$$

The $\mathbb{Q}$-points on $X(\mathrm{e} 7)$ are $(-1 / 3, \pm 14 / 9)$. The Mordell-Weil group over $\mathbb{Q}$ is given by

$$
\frac{\mathbb{Z}}{2 \mathbb{Z}} \cdot[(-1 / 3,-14 / 9)-(-1 / 3,14 / 9)]
$$

\section{Remarks.}

- The models given for $X(\mathrm{~d} 7)$ and $X(\mathrm{e} 7)$ are 'quartic' genus 1 curves. As both have rational points, we can also give Weierstrass models. In fact, a Weierstrass model for $X(\mathrm{~d} 7)$ is

$$
y^{2}+x y=x^{3}-x^{2}-107 x+552
$$

with Cremona label 49A3. A Weierstrass model for $X(\mathrm{e} 7)$ is

$$
y^{2}+x y=x^{3}-x^{2}-1822 x+30393
$$


with Cremona label 49A4. We prefer to work with the models in the lemma, as the $j$-maps are expressible simply in terms of the $x$-coordinates, a fact that is crucial for the proof of Lemma 17.1.

- A model for $X(\mathrm{~d} 7)$ together with the $j$-map has been computed by Elkies and is quoted by Sutherland [77, Section 3]. Elkies' model and $j$-map are equivalent to ours.

Proof of Lemma 15.2. Recall that $C_{\mathrm{s}}^{+}(7)$ and $C_{\mathrm{ns}}^{+}(7)$ respectively denote the normalizers of the split and non-split Cartan subgroups in $\mathrm{GL}_{2}\left(\mathbb{F}_{7}\right)$. By definition, curves $X(\mathrm{~d} 7)$ and $X(\mathrm{e} 7)$ are in fact $X\left(H_{1}\right)$ and $X\left(H_{2}\right)$ where $H_{1}, H_{2}$ are as in Proposition 9.1. Then $H_{i} \cap \mathrm{SL}_{2}\left(\mathbb{F}_{7}\right)$ with $i=1,2$ is a subgroup of index 2 respectively in $C_{\mathrm{s}}^{+}(7) \cap \mathrm{SL}_{2}\left(\mathbb{F}_{7}\right)$ and $C_{\mathrm{ns}}^{+}(7) \cap \mathrm{SL}_{2}\left(\mathbb{F}_{7}\right)$. Thus the inclusion $H_{2} \subset C_{\mathrm{ns}}^{+}(7)$ induces the following commutative diagram:

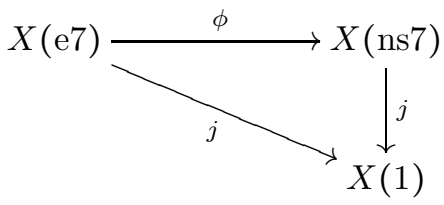

where $\phi$ has degree 2 . We also obtain a similar diagram in which $X(\mathrm{~d} 7)$ and $X(\mathrm{~s} 7)$ respectively replace $X(\mathrm{e} 7)$ and $X(\mathrm{~ns} 7)$. The curves $X(\mathrm{~s} 7)$ and $X(\mathrm{~ns} 7)$ are rational. In [14, Section 5.1], Chen derives expressions for the maps $j: X(\mathrm{~s} 7) \rightarrow X(1)$ and $j: X(\mathrm{~ns} 7) \rightarrow X(1)$. These are given above in (13) and (14) where $x$ is respectively a Hauptmodul for $X(\mathrm{~s} 7), X(\mathrm{~ns} 7)$. We used these to derive the models given for $X(\mathrm{~d} 7)$ and $X(\mathrm{e} 7)$. We only give the details for $X(\mathrm{e} 7)$; the details for $X(\mathrm{~d} 7)$ are an easy adaptation of what follows.

From (14), the map $X(\mathrm{~ns} 7) \rightarrow X(1)$ has degree 21. Subtracting 1728 from both sides of (14) and factoring gives

$$
j-1728=\frac{\left(16 x^{4}+68 x^{3}+111 x^{2}+62 x+11\right) \cdot G(x)^{2}}{\left(x^{3}+x^{2}-2 x-1\right)^{7}},
$$

where

$$
G(x)=7\left(9 x^{4}+26 x^{3}+34 x^{2}+20 x+4\right)\left(x^{4}+6 x^{3}+17 x^{2}+10 x+2\right) .
$$

It is convenient to view both $j: X(\mathrm{~ns} 7) \rightarrow X(1)$ and $j: X(\mathrm{e} 7) \rightarrow X(1)$ as Belyi covers branched at 0,1728 and $\infty$. The factorizations in (14) and (15) yield the ramification data for $X(\mathrm{~ns} 7) \rightarrow X(1)$ :

- there are 7 points above 0 , say $p_{1}, \ldots, p_{7}$, all having ramification degree 3 ;

- there are 13 points above 1728 , say $q_{1}, \ldots, q_{13}$, with $q_{1}, \ldots, q_{5}$ unramified and $q_{6}, \ldots, q_{13}$ having ramification degree 2

- there are 3 points above $\infty$, say $r_{1}, r_{2}, r_{3}$, all having ramification degree 7 . The corresponding ramification data for $X(\mathrm{e} 7) \rightarrow X(1)$ can be deduced from the group $\mathrm{H}_{2}$; for this we applied the formulae of Ligozat [50, which give the following information:

- there are 14 points above 0 , say $P_{1}, \ldots, P_{14}$, all having ramification degree 3 ;

- there are 22 points above 1728 , say $Q_{1}, \ldots, Q_{22}$, with $Q_{1}, Q_{2}$ unramified, and $Q_{3}, \ldots, Q_{22}$ having ramification degree 2 ; 
- there are 6 points above $\infty$, say $R_{1}, \ldots, R_{6}$, all having ramification degree 7 .

It is clear from the above data that the degree 2 cover $\phi: X(\mathrm{e} 7) \rightarrow X(\mathrm{~ns} 7)$ is branched precisely at four of the five points $q_{1}, \ldots, q_{5}$ and nowhere else. One of these five points is $\infty \in X(\mathrm{~ns} 7)(\mathbb{Q})$ and the other four correspond to the roots of the irreducible factor $16 x^{4}+68 x^{3}+111 x^{2}+62 x+11$ in (15). Since the set of branch points is stable under the action of Galois, we see that $\phi$ is branched precisely at the roots of $16 x^{4}+68 x^{3}+111 x^{2}+62 x+11$. It follows that $X(\mathrm{e} 7)$ has an equation of the form

$$
D_{c}: y^{2}=c\left(16 x^{4}+68 x^{3}+111 x^{2}+62 x+11\right),
$$

where $c$ is a non-zero, squarefree integer. The map $\phi$ is then given by $(x, y) \mapsto x$, and the map $j: X(\mathrm{e} 7) \rightarrow X(1)$ is given by the expression in (14). As $H_{2}$ is a congruence group of level 7 , the modular curve $X(\mathrm{e} 7)$ has a model over $\operatorname{Spec}(\mathbb{Z}[1 / 7])$, and so its Jacobian elliptic curve has good reduction outside 7. Using the formulae in [32, the Jacobian elliptic curve of $D_{c}$ is given by

$$
E_{c}: Y^{2}=X^{3}+111 c X^{2}+3512 c^{2} x+34224 .
$$

The model $E_{c}$ has discriminant $2^{12} \cdot 7^{3} \cdot c^{6}$. If $p \mid c$ is a prime $\neq 7$ then the minimal model for $E_{c}$ has bad reduction at $p$. Thus $c$ is not divisible by any prime $p \neq 7$. It follows that $c= \pm 1, \pm 7$. We checked that the only values for which the minimal model of $E_{c}$ has good reduction at 2 are $c=-1$ and $c=7$. Thus one of the two curves $D_{-1}, D_{7}$ is a model for $X(\mathrm{e} 7)$. The curve $D_{-1}$ has no real points. It is however easy to see that $X(\mathrm{e} 7)$ must have real points. For example if $E / \mathbb{R}$ is any real elliptic curve, then the image of $\bar{\rho}_{E / \mathbb{R}, 7}$ has order 2 and every subgroup of $\mathrm{GL}_{2}\left(\mathbb{F}_{7}\right)$ of order 2 is conjugate to a subgroup of $H_{2}$, thus $E / \mathbb{R}$ gives rise to a real point on $X(\mathrm{e} 7)$. It follows that $D_{7}$ is a model for $X(\mathrm{e} 7)$.

This model has two obvious rational points $(-1 / 3, \pm 14 / 9)$ (both with $j$-invariant $0)$. Taking $X(\mathrm{e} 7)$ into Weierstrass form gives the elliptic curve with Cremona label $49 \mathrm{~A} 4$, which has Mordell-Weil group isomorphic to $\mathbb{Z} / 2 \mathbb{Z}$. Thus $(-1 / 3, \pm 14 / 9)$ are all the rational points of $X(\mathrm{e} 7)$. Finally the divisor $(-1 / 3,-14 / 9)-(-1 / 3,14 / 9)$ cannot be principal as $X(\mathrm{e} 7)$ has genus 1 , and so no two points are linearly equivalent. Hence it must generate $\mathrm{Pic}^{0} X(\mathrm{e} 7)$.

15.2. The Modular Curves $X(\mathrm{~b} 3, \mathrm{~b} 5)$ and $X(\mathrm{~s} 3, \mathrm{~b} 5)$. In Lemmas 15.3 and 15.4 below we give models for $X(\mathrm{~b} 3, \mathrm{~b} 5)$ and $X(\mathrm{~s} 3, \mathrm{~b} 5)$ as well as their rational points and the Mordell-Weil groups of their Jacobians. In fact most of this is known (for example [63, Section 2]) but we know of no reference for the $j$-maps, which are essential for our later proofs. The method of Galbraith (sketched in the proof of Lemma 13.1) applies only to non-hyperelliptic modular curves of genus $\geq 3$, for which weight 2 cusp forms can be used to define an embedding into projective space. We now sketch an alternative based on a paper of Muić [57, Theorem 3$3]$. Let $X$ be a modular curve of genus $g$ corresponding to a congruence subgroup $\Gamma \subseteq \mathrm{SL}_{2}(\mathbb{Z})$. Let $k \geq 3$ and let $S_{k}(\Gamma)$ be the space of cusp forms for $\Gamma$ of weight $k$. Write $t$ for the dimension $S_{k}(\Gamma)$, and let $f_{1}, \ldots, f_{t}$ be a basis. If $t \geq g+2$ then $f_{1}, \ldots, f_{t}$ define an embedding $|\mathfrak{c}|$ of $X$ into $\mathbb{P}^{t-1}$ given by a very ample divisor $\mathfrak{c}$ of degree $t+g-1$. In particular, if $t \geq g+3$, and so $\operatorname{deg}(\mathfrak{c}) \geq 2 g+2$, the image of the embedding is a scheme-theoretic intersection of quadrics [22, page 130]. By using higher weight cusp forms where the space has sufficiently large dimension one can 
straightforwardly adapt the method sketched in the proof of Lemma 13.1 to write down a projective model for $X$ and the associated $j$-map $X \rightarrow X(1)$.

For $X(\mathrm{~b} 3, \mathrm{~b} 5)$ and $X(\mathrm{~s} 3, \mathrm{~b} 5)$ the spaces of cusp forms of weight 4 have dimensions 4 and 8 respectively, and this allowed us to write models for these curves as intersections of quadrics in $\mathbb{P}^{3}$ and $\mathbb{P}^{7}$ respectively, as well as expressions for the $j$-maps. Transforming these into Weierstrass models gives Lemmas 15.3 and 15.4 below; the expressions for the $j$-maps on these two curves are complicated but can be found in the accompanying Magma script (see page 5).

Lemma 15.3. The curve $X(\mathrm{~b} 3, \mathrm{~b} 5)=X_{0}(15)$ is an elliptic curve with the Weierstrass model

$$
X(\mathrm{~b} 3, \mathrm{~b} 5): y^{2}+x y+y=x^{3}+x^{2}-10 x-10
$$

and Cremona label 15A1. Its $\mathbb{Q}$-points are

$$
\infty,(-1,0),(-2,-2),(8,-27),(3,-2),(-13 / 4,9 / 8),(-2,3),(8,18) .
$$

The Mordell-Weil group over $\mathbb{Q}$ is

$$
\frac{\mathbb{Z}}{4 \mathbb{Z}} \cdot[(-1,0)-\infty] \oplus \frac{\mathbb{Z}}{2 \mathbb{Z}}[(-2,-2)-\infty] .
$$

Lemma 15.4. The curve $X(\mathrm{~s} 3, \mathrm{~b} 5)$ is an elliptic curve with the Weierstrass model

$$
X(\mathrm{~s} 3, \mathrm{~b} 5): y^{2}+x y+y=x^{3}+x^{2}-5 x+2,
$$

and Cremona label 15A3. The $\mathbb{Q}$-points of this model are

$$
\infty,(3 / 4,-7 / 8),(0,-2),(2,-4),(1,-1),(-3,1),(0,1),(2,1) .
$$

The Mordell-Weil group of the Jacobian over $\mathbb{Q}$ is

$$
\frac{\mathbb{Z}}{4 \mathbb{Z}} \cdot[(0,-2)-\infty] \oplus \frac{\mathbb{Z}}{2 \mathbb{Z}} \cdot[(3 / 4,-7 / 8)-\infty] .
$$

\section{A Sieve for the Symmetric Square of a Fibre Product}

Let $X_{1}, X_{2}$ be curves of genus $\geq 1$ defined over $\mathbb{Q}$, and let $J_{1}, J_{2}$ be their Jacobians. Let $\mu_{k}: X_{k} \rightarrow \mathbb{P}^{1}$ be morphisms defined over $\mathbb{Q}$ for $k=1$, 2. Suppose that the fibre product $X_{1} \times_{\mathbb{P}^{1}} X_{2}$ is irreducible and let $X$ be its normalization. Let $\pi_{k}: X \rightarrow X_{k}$ be the induced projection maps. Fix rational degree 2 divisors $D_{k}$ on $X_{k}$ and let

$$
\iota_{k}: X_{k}^{(2)} \rightarrow J_{k}, \quad D \mapsto\left[D-D_{k}\right]
$$

be the corresponding Abel-Jacobi maps for the symmetric squares. Let

$$
\alpha: X^{(2)} \rightarrow J_{1} \times J_{2}, \quad D \mapsto\left(\iota_{1}\left(\pi_{1} D\right), \iota_{2}\left(\pi_{2} D\right)\right) .
$$

Let $p_{1}, \ldots, p_{r}$ be primes of good reduction for $X$. For each $p=p_{i}$ we have the commutative diagram

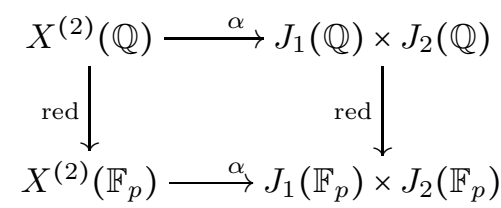

where the vertical maps denote reduction modulo $p$. The following lemma is an obvious consequence of the diagram's commutativity. 
Lemma 16.1. With the above notation and assumptions,

$$
\alpha\left(X^{(2)}(\mathbb{Q})\right) \subseteq \bigcap_{p=p_{i}} \operatorname{red}^{-1} \alpha\left(X^{(2)}\left(\mathbb{F}_{p}\right)\right) .
$$

In particular, if the intersection on the right-hand side is empty, then $X^{(2)}(\mathbb{Q})=\varnothing$ and so the curve $X$ does not have any rational or quadratic points.

Remark. Let $X / \mathbb{Q}$ be a curve of genus $\geq 2$ and let $J$ be its Jacobian. Fix some $P_{0} \in X(\mathbb{Q})$ and let $\iota: X \rightarrow J$ be the corresponding Abel-Jacobi map $P \mapsto\left[P-P_{0}\right]$. For any prime $p$ of good reduction for $X$, we have a commutative diagram

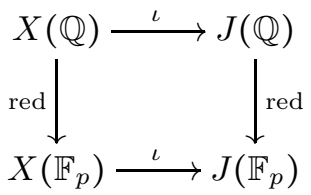

If $p_{1}, \ldots, p_{r}$ we have

$$
\iota(X(\mathbb{Q})) \subseteq \bigcap_{p=p_{i}} \operatorname{red}^{-1} \iota\left(X\left(\mathbb{F}_{p}\right)\right) .
$$

This sieve is known as the Mordell-Weil sieve (e.g. [76, Section 3]) and gives information on the image of $X(\mathbb{Q})$ inside $J(\mathbb{Q})$. The Mordell-Weil sieve can be extended in the obvious manner to the symmetric square of $X$ (see for example [69, Section 5]), and then it gives information on the image of $X^{(2)}(\mathbb{Q})$ inside $J(\mathbb{Q})$. Now suppose $X$ is the normalization of a fibre product $X_{1} \times_{\mathbb{P}^{1}} X_{2}$ as above. The Jacobian $J$ is in general much larger (in dimension) than $J_{1} \times J_{2}$, and the information provided by Lemma 16.1 will be weaker than that given by the Mordell-Weil sieve. However, in the Section [17 where we apply Lemma 16.1, it is realistic to carry out explicit computations with $J_{1}, J_{2}$ but not with $J$. Indeed, in our applications $J_{1}$ and $J_{2}$ will be elliptic curves, whereas the possible dimensions for $J$ will be 97,153 , 73 and 113.

16.1. Points on the Normalization of a Fibre Product. To apply Lemma16.1, we need to enumerate the points of $X^{(2)}\left(\mathbb{F}_{p}\right)$ for several primes $p$. To enumerate $X^{(2)}\left(\mathbb{F}_{p}\right)$ it is enough to know the points of $X\left(\mathbb{F}_{p}\right)$ and $X\left(\mathbb{F}_{p^{2}}\right)$, and this is easy in practice if $p$ is sufficiently small and one has a convenient model (say smooth and projective) for $X$. For the four curves (3) we will apply the lemma to, it seems impractical to write down such a model in view of the very large genera of these curves (Lemma 15.1). In what follows we will use a description of the points of the normalization $X$ of $X_{1} \times_{\mathbb{P}^{1}} X_{2}$ in terms of the points of $X_{1}, X_{2}$. This will allow us to enumerate the points of $X$ over finite fields.

We continue with the above notation, and we let $\mathbb{F}$ be either an extension of $\mathbb{Q}$ or an extension of $\mathbb{F}_{p}$ where $p$ is a prime of good reduction for $X, X_{1}, X_{2}$ and the projection maps, and such that the characteristic of $\mathbb{F}$ does not divide the ramification indices of the maps $\mu_{k}$. By definition of $X_{1} \times_{\mathbb{P}^{1}} X_{2}$, if $P \in X(\mathbb{F})$, and $P_{k}=\pi_{k}(P)$ for $k=1,2$ then $P_{k} \in X_{k}(\mathbb{F})$ and $\mu_{1}\left(P_{1}\right)=\mu_{2}\left(P_{2}\right)$. Now suppose $P_{1} \in X_{1}(\mathbb{F}), P_{2} \in X_{2}(\mathbb{F})$ satisfy $\mu_{1}\left(P_{1}\right)=\mu_{2}\left(P_{2}\right)$. We would like to describe the points $P \in X(\mathbb{F})$ such that $P_{k}=\pi_{k}(P)$ for $k=1,2$. Here we follow the description of Flynn and Testa [33, Section 2]. Write $\gamma=\mu_{1}\left(P_{1}\right)=\mu_{2}\left(P_{2}\right) \in \mathbb{P}^{1}(\mathbb{F})$. Suppose 
first that $\gamma \neq \infty$, so we may think of $\gamma \in \mathbb{A}^{1}(\mathbb{F})$. We consider $\mu_{1}, \mu_{2}$ as elements of the completions of the local rings of $X_{k}$ at $P_{k}$, which we denote by $\widehat{\mathcal{O}_{k}}$. Then

$$
\mu_{1}=\gamma+\epsilon s^{m}, \quad \mu_{2}=\gamma+\delta t^{n}
$$

where $s \in \widehat{\mathcal{O}_{1}}, t \in \widehat{\mathcal{O}_{2}}$ are elements of valuation 1 , the coefficients $\epsilon$ and $\delta$ belong to $\mathbb{F}^{*}$, and $m, n$ are respectively the ramification indices for $\mu_{1}, \mu_{2}$ at $P_{1}, P_{2}$. A local equation for $X_{1} \times_{\mathbb{P} 1} X_{2}$ in the neighbourhood of $\left(P_{1}, P_{2}\right)$ is given by

$$
\epsilon s^{m}=\delta t^{n} \text {. }
$$

Of course, on this local equation, the point $\left(P_{1}, P_{2}\right)$ is represented by $(0,0)$. Now let $d=\operatorname{gcd}(m, n)$. Over $\overline{\mathbb{F}}$, the local equation has $d$ components: $\lambda s^{m^{\prime}}=t^{n^{\prime}}$ where $m^{\prime}=m / d, n^{\prime}=n / d$ and $\lambda$ runs through the $d$-th roots of $\epsilon / \delta$. We normalize these components by letting $N=m^{\prime} \cdot n^{\prime}$ and making the substitutions

$$
s=\lambda^{a} r^{n^{\prime}}, \quad t=\lambda^{b} r^{m^{\prime}},
$$

with $a, b \in \mathbb{Z}$ chosen so that $a m^{\prime}-b n^{\prime}=-1$. It follows that there are precisely $d$ points $P \in X$ above $\left(P_{1}, P_{2}\right)$. We may represent these by $\left(P_{1}, P_{2}, \lambda\right)$ where $\lambda^{d}=\epsilon / \delta$. Such a point belongs to $X(\mathbb{F})$ if and only if $P_{1} \in X_{1}(\mathbb{F}), P_{2} \in X_{2}(\mathbb{F})$ and $\lambda \in \mathbb{F}$. Moreover, the parameter $r$ has valuation 1 in the completion of the local ring of $X$ at $P$. The ramification index for $X \rightarrow \mathbb{P}^{1}$ at each of these $P$ is $m n^{\prime}=m^{\prime} n=m n / \operatorname{gcd}(m, n)$. To see this, note that the composition of maps

$$
X \rightarrow X_{1} \times_{\mathbb{P}^{1}} X_{2} \rightarrow X_{1} \rightarrow \mathbb{P}^{1}
$$

viewed as an element of the completion of the local ring of $X$ at $P$ can be written as

$$
\mu_{1}=\gamma+\epsilon s^{m}=\gamma+\epsilon\left(\lambda^{a} r^{n^{\prime}}\right)^{m}=\gamma+\epsilon \cdot \lambda^{a} \cdot r^{m n^{\prime}} .
$$

The above argument needs an easy adaptation if $\gamma=\infty \in \mathbb{P}^{1}$. When representing the points of $X$ as $P=\left(P_{1}, P_{2}, \lambda\right)$ the projection maps are obvious: $\pi_{k}(P)=P_{k}$.

If $\mathbb{F}=\mathbb{F}_{p}$ or $\mathbb{F}_{p^{2}}$, the above gives an algorithm for constructing the $\mathbb{F}$-points of $X$ from the $\mathbb{F}$-points of $X_{1}, X_{2}$ and so it enables us to write down $X^{(2)}\left(\mathbb{F}_{p}\right)$.

17. Real Quadratic Points on $X(\mathrm{~b} 3, \mathrm{~b} 5, \mathrm{~d} 7), X(\mathrm{~s} 3, \mathrm{~b} 5, \mathrm{~d} 7), X(\mathrm{~b} 3, \mathrm{~b} 5, \mathrm{e} 7)$, AND $X(\mathrm{~s} 3, \mathrm{~b} 5, \mathrm{e} 7)$.

Lemma 17.1. The curve $X(\mathrm{~b} 3, \mathrm{~b} 5, \mathrm{e} 7)$ does not have any rational or quadratic points. The non-cuspidal real quadratic points on the curves

$$
X(\mathrm{~b} 3, \mathrm{~b} 5, \mathrm{~d} 7), \quad X(\mathrm{~s} 3, \mathrm{~b} 5, \mathrm{~d} 7), \quad X(\mathrm{~s} 3, \mathrm{~b} 5, \mathrm{e} 7)
$$

are modular.

Proof. Let $X=X(\mathrm{~b} 3, \mathrm{~b} 5, \mathrm{~d} 7)$ which we think of as the normalization of $X_{1} \times \mathbb{P}^{1} X_{2}$ where $X_{1}=X(\mathrm{~b} 3, \mathrm{~b} 5), X_{2}=X(\mathrm{~d} 7)$ with the maps $\mu_{k}: X_{k} \rightarrow \mathbb{P}^{1}$ being just the $j$-maps to $X(1)$. Write $J_{1}, J_{2}$ for the Jacobians of $X_{1}, X_{2}$. In Section 15 we determined models for the genus 1 curves $X_{1}, X_{2}$ as well as the Mordell-Weil groups $J_{1}(\mathbb{Q}), J_{2}(\mathbb{Q})$. We fix degree 2 divisors

$$
D_{1}=2 \infty, \quad D_{2}=2 \cdot(5 / 2,7 / 4)
$$

on $X_{1}, X_{2}$ respectively, which we use to define Abel-Jacobi maps $X_{k}^{(2)} \rightarrow J_{k}$ as in (17). We now apply Lemma 16.1 with $11 \leq p<100$. We note that these primes are 
of good reduction for $X$, and that they do not divide the ramification indices for the maps $X_{k} \rightarrow X(1)$. We find that

$$
\begin{aligned}
\alpha\left(X^{(2)}(\mathbb{Q})\right) \subseteq\{ & ([0],[(5 / 2,-7 / 4)-(5 / 2,7 / 4)]), \\
& ([(3,-2)-\infty],[(5 / 2,-7 / 4)-(5 / 2,7 / 4)]), \\
& ([(8,18)-\infty],[(5 / 2,-7 / 4)-(5 / 2,7 / 4)])\} \\
\subseteq & J_{1}(\mathbb{Q}) \times J_{2}(\mathbb{Q}) .
\end{aligned}
$$

Now let $P$ be a quadratic point on $X$ and let $P^{\sigma}$ be its conjugate. Then $D=$ $P+P^{\sigma} \in X^{(2)}(\mathbb{Q})$. From (20) and the definition of $\alpha$, we have

$$
\pi_{2}(P)+\pi_{2}\left(P^{\sigma}\right)-D_{2} \sim(5 / 2,-7 / 4)-(5 / 2,7 / 4) .
$$

Thus

$$
\pi_{2}(P)+\pi_{2}\left(P^{\sigma}\right)=\operatorname{div}(f)+(5 / 2,7 / 4)+(5 / 2,-7 / 4)
$$

for some non-zero function $f$ on $X_{2}=X(\mathrm{~d} 7)$. The function $f$ belongs to the Riemann-Roch space of $(5 / 2,7 / 4)+(5 / 2,-7 / 4)$. A $\mathbb{Q}$-basis for this Riemann-Roch space is

Thus

$$
\frac{1}{2 x-5}, \quad \frac{x}{2 x-5} \text {. }
$$

$$
f=\frac{a+b x}{2 x-5}
$$

for some $a, b \in \mathbb{Q}$, not both zero. Thus the $x$-coordinate of $\pi_{2}(P)$ and $\pi_{2}\left(P^{\sigma}\right)$ is $-a / b \in \mathbb{P}^{1}(\mathbb{Q})$. From Lemma 15.2 , we know that the $j$-value of $\pi_{2}(P)$ is expressible in terms of its $x$-coordinate as given by $(13)$, hence it belongs to $\mathbb{P}^{1}(\mathbb{Q})$. Now suppose $P$ is a non-cuspidal real quadratic point on $X$. It follows that any elliptic curve supporting $P$ has $j$-invariant belonging to $\mathbb{Q}$ and so is modular by $[9]$.

We apply the same sieve to $X=X(\mathrm{~s} 3, \mathrm{~b} 5, \mathrm{~d} 7)$, where we take $X_{1}=X(\mathrm{~s} 3, \mathrm{~b} 5)$ and $X_{2}=X(\mathrm{~d} 7)$, and we take $D_{1}, D_{2}$ as in (19), where of course, now $\infty$ is the point at infinity on the model for $X_{1}$ given in (16). We obtained

$$
\begin{aligned}
\alpha\left(X^{(2)}(\mathbb{Q})\right) \subseteq\{ & ([0],[(5 / 2,-7 / 4)-(5 / 2,7 / 4)]), \\
& ([(2,-4)-\infty],[(5 / 2,-7 / 4)-(5 / 2,7 / 4)]), \\
& ([(1,-1)-\infty],[(5 / 2,-7 / 4)-(5 / 2,7 / 4)])\} \\
\subseteq & J_{1}(\mathbb{Q}) \times J_{2}(\mathbb{Q}) .
\end{aligned}
$$

By exactly the same argument as before, quadratic points have rational $j$-invariants, and thus non-cuspidal real quadratic points are modular.

Applying the sieve to $X=X(\mathrm{~b} 3, \mathrm{~b} 5, \mathrm{e} 7)$ shows that $\alpha\left(X^{(2)}(\mathbb{Q})\right)$ is empty, so these this curve have no rational or quadratic points.

Finally, we apply the sieve to $X=X(\mathrm{~s} 3, \mathrm{~b} 5, \mathrm{e} 7)$. Of course, we take $X_{1}=$ $X(\mathrm{~s} 3, \mathrm{~b} 5), X_{2}=X(\mathrm{e} 7)$. We fix the degree 2 divisors

$$
D_{1}=2 \infty, \quad D_{2}=2 \cdot(-1 / 3,14 / 9)
$$


of $X_{1}$ and $X_{2}$. The sieve yields

$$
\alpha\left(X^{(2)}(\mathbb{Q})\right) \subseteq\{([(0,1)-\infty],[(-1 / 3,-14 / 9)-(-1 / 3,14 / 9)])\} .
$$

If $P$ is a quadratic point on $X$ and $\pi_{2}(P)$ is its image on $X_{2}=X(\mathrm{e} 7)$ then

$$
\pi_{2}(P)+\pi_{2}\left(P^{\sigma}\right)=\operatorname{div}(f)+(-1 / 3,14 / 9)+(-1 / 3,-14 / 9) .
$$

As before, the $x$-coordinate of $\pi_{2}(P)$ on the model for $X(\mathrm{e} 7)$ belongs to $\mathbb{P}^{1}(\mathbb{Q})$ and so the $j$-invariant (given by (14) ) is rational. This completes the proof.

\section{Remarks.}

(I) In the above, we have morphisms $\alpha: X^{(2)} \rightarrow J_{1} \times J_{2}$. The points $X^{(2)}(\mathbb{Q})$ belong to the fibres $\alpha^{-1}\left(J_{1}(\mathbb{Q}) \times J_{2}(\mathbb{Q})\right)$. Since $J_{1}(\mathbb{Q})$ and $J_{2}(\mathbb{Q})$ are finite and explicitly known, the reader is perhaps puzzled as to why we do not use this to recover $X^{(2)}(\mathbb{Q})$. We are in fact able to write down equations for these fibres as schemes in $\mathbb{P}^{1} \times \mathbb{P}^{1}$. Most of these are zero-dimensional, but are of such high degree that we have found it impractical to decompose them using Gröbner basis computations. A few of the fibres are however 1-dimensional. These are so complicated that we are unable to say anything about their rational points.

(II) Our sieve cannot prove that $X^{(2)}(\mathbb{Q})$ is empty for the three modular curves (18). It is natural to ask whether these curves have rational or quadratic points. The genera of these three modular curves are 97, 153 and 113 respectively by Lemma 15.1. As these are so large, it is reasonable to suspect that the only quadratic points lie above 0,1728 or $\infty$ (and Remark (III) below shows that this is indeed the case). Using the description of the points of $X=X(\mathrm{~b} 3, \mathrm{~b} 5, \mathrm{~d} 7)$ as the fibre product of $X_{1}=X(\mathrm{~b} 3, \mathrm{~b} 5)$ and $X_{2}=X(\mathrm{~d} 7)$ we find that there are precisely 8 quadratic cusps, and no quadratic points above 0 and 1728 . We shall denote the quadratic cusps by $P_{1}^{ \pm}, P_{2}^{ \pm}, P_{3}^{ \pm}, P_{4}^{ \pm}$. These respectively lie above

$$
\left(\infty, \infty_{ \pm}\right),\left((-1,0), \infty_{ \pm}\right),\left((-2,3), \infty_{ \pm}\right),\left((8,18), \infty_{ \pm}\right) \in X_{1} \times_{\mathbb{P}^{1}} X_{2} .
$$

Here $\infty_{+}, \infty_{-} \in X_{2}$ are conjugates defined over $\mathbb{Q}(\sqrt{-7})$. Thus $P_{i}^{+}+P_{i}^{-}$belong to $X^{(2)}(\mathbb{Q})$. We find that

$$
\begin{aligned}
& \alpha\left(P_{1}^{+}+P_{1}^{-}\right)=\alpha\left(P_{2}^{+}+P_{2}^{-}\right)=([0],[(5 / 2,-7 / 4)-(5 / 2,7 / 4)]), \\
& \alpha\left(P_{3}^{+}+P_{3}^{-}\right)=\alpha\left(P_{4}^{+}+P_{4}^{-}\right)=([(3,-2)-\infty],[(5 / 2,-7 / 4)-(5 / 2,7 / 4)]) .
\end{aligned}
$$

This is consistent with the result of the sieve given in (20), and provides a useful check on the correctness of our computations.

We carried the same computation for $X=X(\mathrm{~s} 3, \mathrm{~b} 5, \mathrm{~d} 7)$ and found quadratic cusps $P_{1}^{ \pm}, P_{2}^{ \pm}, P_{3}^{ \pm}, P_{4}^{ \pm}$and no other quadratic points above 0,1728 and $\infty$. The four quadratic cusps respectively lie above

$$
\left(\infty, \infty_{ \pm}\right),\left((0,1), \infty_{ \pm}\right),\left((2,-4), \infty_{ \pm}\right),\left((-3,1), \infty_{ \pm}\right) \in X_{1} \times_{\mathbb{P} 1} X_{2},
$$

where of course $X_{1}=X(\mathrm{~s} 3, \mathrm{~b} 5)$ and $X_{2}=X(\mathrm{~d} 7)$. We find that

$$
\begin{aligned}
& \alpha\left(P_{1}^{+}+P_{1}^{-}\right)=\alpha\left(P_{4}^{+}+P_{4}^{-}\right)=([0],[(5 / 2,-7 / 4)-(5 / 2,7 / 4)]), \\
& \alpha\left(P_{2}^{+}+P_{2}^{-}\right)=\alpha\left(P_{3}^{+}+P_{3}^{-}\right)=([(1,-1)-\infty],[(5 / 2,-7 / 4)-(5 / 2,7 / 4)]) .
\end{aligned}
$$

This is consistent with the result of the sieve given in (21). 
As a final check on our computations, we verified that there are no quadratic points on $X(\mathrm{~b} 3, \mathrm{~b} 5, \mathrm{e} 7)$ and $X(\mathrm{~s} 3, \mathrm{~b} 5, \mathrm{e} 7)$ above 0,1728 and $\infty$.

(III) Although we do not need this for the proof of Lemma 17.1, it is interesting to ask if it can be shown that all quadratic points $P$ on $X_{1}:=X(\mathrm{~b} 3, \mathrm{~b} 5, \mathrm{~d} 7), X_{2}:=$ $X(\mathrm{~s} 3, \mathrm{~b} 5, \mathrm{~d} 7), X_{3}:=X(\mathrm{~s} 3, \mathrm{~b} 5, \mathrm{e} 7)$ do satisfy $j(P) \in\{0,1728, \infty\}$. We explain how to solve this problem by reducing it to the determination of rational points on some modular curves with 'milder' level structure. Indeed, let $P$ be a non-cuspidal quadratic point on $X$, where $X$ is one of these three modular curves $X_{1}, X_{2}, X_{3}$. We know by the proof of Lemma 17.1 that $j(P) \in \mathbb{Q}$. Thus the point $P$ is supported by some elliptic curve $E$ defined over $\mathbb{Q}$, even though the level structure is defined over some quadratic field $K$. We know in particular that $\bar{\rho}_{E, 5}\left(G_{K}\right)$ is contained in a Borel subgroup $B(5)$ of $\mathrm{GL}_{2}\left(\mathbb{F}_{5}\right)$. Now $\bar{\rho}_{E, 5}\left(G_{K}\right)$ is a subgroup of $\bar{\rho}_{E, 5}\left(G_{\mathbb{Q}}\right)$ of index 1 or 2. By a straightforward analysis of the subgroups of $\mathrm{GL}_{2}\left(\mathbb{F}_{5}\right)$ it follows that $\bar{\rho}_{E, 5}\left(G_{\mathbb{Q}}\right)$ is contained either in $B(5)$ or $C_{\mathrm{s}}^{+}(5)$ (the normalizer of a split Cartan subgroup). Similarly, for $X=X_{1}$, we have $\bar{\rho}_{E, 3}\left(G_{\mathbb{Q}}\right)$ is contained either in $B(3)$ or $C_{\mathrm{s}}^{+}(3)$ and for $X=X_{2}, X=X_{3}$ it is contained in $C_{\mathrm{ns}}^{+}(3)$. Finally, for $X=X_{1}, X_{2}$, the image $\bar{\rho}_{E, 7}\left(G_{\mathbb{Q}}\right)$ is contained in $C_{\mathrm{s}}^{+}(7)$ and for $X=X_{3}$ it is contained in $C_{\mathrm{ns}}^{+}(7)$. It is now not difficult to show in all cases, with the help of the results of this paper and of [49], that $j(E) \in\{0,1728\}$. Consider $X=X_{1}$ for example. Then $E$ gives rise to a $\mathbb{Q}$-point of one of the curves $X(\mathrm{~b} 3, \mathrm{~b} 5), X(\mathrm{~b} 3, \mathrm{~s} 5), X(\mathrm{~s} 3, \mathrm{~b} 5)$ or $X(\mathrm{~s} 3, \mathrm{~s} 5)$. We have already determined the $\mathbb{Q}$-points on these four curves in the proofs of Lemmas 13.1, 14.1, and in Lemmas 15.3, 15.4. Enumerating the $j$-invariants of these points $Q$, we are able to show for $j(Q) \neq 0,1728$ that they do not pull back to non-cuspidal quadratic points on $X(\mathrm{~d} 7)$.

\section{Modularity of Elliptic Curves over $\mathbb{Q}(\sqrt{5})$}

To complete the proof of Theorem 1 we merely have to prove Lemma 1.1, which states that every elliptic curve over $K=\mathbb{Q}(\sqrt{5})$ is modular. Again we reduce this problem to showing that the $K$-points on certain modular curves are in fact modular (in the sense that they correspond to modular elliptic curves). We give fewer details in this section as compared to previous sections. This is because the task is much simpler: determining the points on a curve over one quadratic field is usually easier than determining the points over all real quadratic fields.

Proof of Lemma 1.1, Let $K=\mathbb{Q}(\sqrt{5})$, and let $E$ be an elliptic curve over $K$. We would like to show that $E$ is modular. By Theorems 3 and 4 if $\bar{\rho}_{E, p}\left(G_{K\left(\zeta_{p}\right)}\right)$ is absolutely irreducible with $p=3$ or 7 then $E$ is modular. Thus we may restrict our attention to elliptic curves $E$ such that $\bar{\rho}_{E, p}\left(G_{K\left(\zeta_{p}\right)}\right)$ is simultaneously absolutely reducible for $p=3$ and 7. Now appealing to Corollary 10.1 we see that $E$ gives rise to a $K$-point on one of the following four modular curves

$$
X(\mathrm{~d} 7), \quad X(\mathrm{e} 7), \quad X(\mathrm{~b} 3, \mathrm{~b} 7), \quad X(\mathrm{~s} 3, \mathrm{~b} 7) .
$$

We determined models for the first two curves in Lemma 15.2 and found that they are isomorphic (over $\mathbb{Q}$ ) to elliptic curves $49 \mathrm{~A} 3$ and 49A4 respectively. The third curve is isomorphic (over $\mathbb{Q}$ ) to the elliptic curve $21 \mathrm{~A} 1$. Let $X$ be any of the first three curves. By considering both $X$ and its $\sqrt{5}$-twist it is easy to show that $X(K)=X(\mathbb{Q})$ in all cases. It follows that the $K$-points on the first three curves are modular. 
Finally let $X=X(\mathrm{~s} 3, \mathrm{~b} 7) \cong X_{0}(63) /\left\langle w_{9}\right\rangle$. This curve has the obvious quotient $Y=X_{0}(63) /\left\langle w_{7}, w_{9}\right\rangle$. The curve $Y$ has genus 1 and is in fact isomorphic to the elliptic curve 21A1. By the above, $Y(K)=Y(\mathbb{Q})$. Thus if $P$ is a non-cuspidal $K$-point on $X$ then its image on $Y$ belongs to $Y(\mathbb{Q})$. Let $\sigma$ be the non-trivial automorphism of $K$. Thus $P$ and $P^{\sigma}$ map to the same point on $Y$. Hence $P^{\sigma}=P$ or $P^{\sigma}=w_{7}(P)$. In the former case $P$ is a rational point and hence modular. In the latter case, $P$ corresponds to a $\mathbb{Q}$-curve, and is therefore modular.

\section{Part 6. Further Directions}

\section{On Modularity over Totally Real Fields (Again)}

Most of the theory we have developed in Parts 2, 3 and 4 applies to elliptic curves over arbitrary totally real fields. If one is interested in modularity of elliptic curves over a particular totally real field $K$ then it might be possible to prove this by the methods of this paper, together with explicit methods for computing rational points on curves (e.g. [70, 76]). However, one can considerably simplify this task by imposing suitable restrictions on the field $K$ and elliptic curve $E$.

Theorem 7. Let $p=5$ or 7 . Let $K$ be a totally real field having some unramified place $v$ above $p$. Let $E$ be an elliptic curve semistable at $v$ and suppose $\bar{\rho}_{E, p}$ is irreducible. Then $E$ is modular.

Proof. As $v / p$ is unramified, we have $K \cap \mathbb{Q}\left(\zeta_{p}\right)=\mathbb{Q}$. Let $I_{v}$ be the inertia subgroup of $G_{K}$ at $v$. By [64, Propositions 11, 12,13], the image of $\bar{\rho}_{E, p}\left(I_{v}\right)$ in $\operatorname{PGL}_{2}\left(\mathbb{F}_{p}\right)$ contains a cyclic subgroup of order $p-1$ or $p+1$. It follows from Proposition 9.1 that $\bar{\rho}\left(G_{K\left(\zeta_{p}\right)}\right)$ is absolutely irreducible. Thus $E$ is modular by Theorems 3 and 4 .

In [31, the first-named author proves a version of Theorem 7 with $p=3$ (and without the irreducibility assumption) using modularity lifting theorems due to Skinner and Wiles [72, 73].

\section{Diophantine Applications}

Wiles' proof of Fermat's Last Theorem provides a template for attacking many other Diophantine equations via Frey curves and modularity (see [6], 68 for recent surveys). Modularity results over totally real fields extend the applicability of this template, as for example in [26. We expect the results and methods of the current paper to play an important rôle in the development of this area. Indeed, a forthcoming paper [34] uses Theorem 1 to study the Fermat equation over real quadratic fields. In particular, it is shown that if $d>6$ is a squarefree integer satisfying $d \equiv 3$ $(\bmod 8)$ or $d \equiv 6,10(\bmod 16)$, then there is an effectively computable constant $B_{d}$ such that for all primes $p>B_{d}$, every solution to the Fermat equation

$$
x^{p}+y^{p}=z^{p}, \quad x, y, z \in \mathbb{Q}(\sqrt{d})
$$

satisfies $x y z=0$.

\section{REFERENCES}

[1] P. B. Allen, Modularity of nearly ordinary 2-adic residually dihedral Galois representations, arXiv:1301.1113v2, 11 September 2013.

[2] B. S. Banwait and J. Cremona, Tetrahedral elliptic curves and the local-to-global principle for isogenies, arXiv:1306.6818v2, 16 August 2013. 
[3] T. Barnet-Lamb, T. Gee and D. Geraghty, Congruences between Hilbert modular forms: constructing ordinary lifts, Duke Math. Journal 161 (2012), 1521-1580.

[4] T. Barnet-Lamb, T. Gee and D. Geraghty, Congruences between Hilbert modular forms: constructing ordinary lifts II, Mathematical Research Letters 20 (2013), 81-86.

[5] F. Bars, A. Kontogeorgis and X. Xarles, Bielliptic and hyperelliptic modular curves $X(n)$ and the group $\operatorname{Aut}(X(n))$, Acta Arithmetica, to appear.

[6] M. A. Bennett, I. Chen, S. R. Dahmen and S. Yazdani, Generalized Fermat equations: a miscellany, preprint, 2013.

[7] W. Bosma, J. Cannon and C. Playoust: The Magma Algebra System I: The User Language, J. Symb. Comp. 24 (1997), 235-265. (See also http://magma.maths.usyd.edu.au/magma/)

[8] D. Blasius and J. D. Rogawski, Motives for Hilbert modular forms, Inventiones Mathematicae 114 (1993), no. 1, 55-87.

[9] C. Breuil, B. Conrad, F. Diamond, R. Taylor, On the modularity of elliptic curves over $\mathbb{Q}$ : wild 3-adic exercises, Journal of the American Mathematical Society 14 (2001), 843-939.

[10] C. Breuil and F. Diamond Formes modulaires de Hilbert modulo $p$ et valeurs d'extensions galoisiennes, Annales Scientifiques de l'École Normale Supérieure, to appear.

[11] P. Bruin and F. Najman, On isogenies of elliptic curves over quadratic fields, preprint, 2013.

[12] H. Carayol, Sur les représentations $\ell$-adiques associées aux formes modulaires de Hilbert, Ann. Sci. Ec. Norm. Sup. 4 (1986), 409-468.

[13] H. Carayol, Sur les représentations galoisiennes modulo attachées aux formes modulaires, Duke Math. J. 59 (1989), 785-801.

[14] I. Chen, The Jacobian of Modular Curves Associated to Cartan Subgroups, DPhil thesis, University of Oxford, 1996.

[15] J. Coates and S. T. Yau (editors), Elliptic Curves, Modular Forms 85 Fermat's Last Theorem, second edition, International Press, Cambridge, MA, 1997.

[16] H. Cohen, Number Theory, Volume II: Analytic and Modern Tools, GTM 240, SpringerVerlag, 2007.

[17] B. Conrad, F. Diamond and R. Taylor, Modularity of certain potentially Basotti-Tate Galois representations, Journal of the American Mathematical Society 12 (1999), no. 2, 521-567.

[18] G. Cornell and J. H. Silverman (editors), Arithmetic Geometry, Springer-Verlag, 1986.

[19] G. Cornell, J. H. Silverman and G. Stevens (editors), Modular Forms and Fermat's Last Theorem, Springer-Verlag, 1997.

[20] J. E. Cremona, Algorithms for Modular Elliptic Curves, Cambridge University Press, 1992.

[21] J. Cremona, J.-C. Lario, J. Quer, K. Ribet (editors), Modular Curves and Abelian Varieties, Progr. Math. 224, Birkhäuser, Basel, 2004.

[22] V. I. Danilov and V. V. Shokurov, Algebraic Curves, Algebraic Manifolds and Schemes, Springer-Verlag, 1998.

[23] H. Darmon, F. Diamond, and R. Taylor, Fermat's last theorem, pages 2-140 of [15].

[24] F. Diamond, On deformation rings and Hecke rings, Annals of Mathematics 144 (1996), $137-166$.

[25] F. Diamond and J. Shurman, A First Course on Modular Forms, GTM 228, Springer, 2005.

[26] L. Dieulefait and N. Freitas Fermat-type equations of signature $(13,13, p)$ via Hilbert cuspforms, Math. Annalen, 2013, (to appear).

[27] J. Ellenberg Serre's conjecture over $\mathbb{F}_{9}$, Ann. of Math. (2) 161 (2005), 1111-1142.

[28] N. Elkies, The Klein quartic in number theory, in The Eightfold Way: The Beauty of Klein's Quartic Curve, ed. Silvio Levy, MSRI Publications Volume 35, 1998.

[29] G. Faltings, Endlichkeitssätze für abelsche Varietäten über Zahlkörpern, Invent. Math. 73 (1983), no. 3, 349-366.

[30] G. Faltings, The general case of S. Langs conjecture, pages 175182 of Barsotti Symposium in Algebraic Geometry (Abano Terme, 1991), Perspect. Math. 15, Academic Press, San Diego, CA, 1994.

[31] N. Freitas, Recipes for Fermat-type equations of the form $x^{r}+y^{r}=C z^{p}$,

[32] T. Fisher, The invariants of a genus one curves, Proc. London Math. Soc. 97 (2008), 753782.

[33] E. V. Flynn and D. Testa, Finite Weil restrictions of curves, arXiv:1210.4407 $\mathrm{J} 3,19$ May 2013.

[34] N. Freitas and S. Siksek, Modularity and the Fermat equation over totally real fields, arXiv:1307.3162, 11 July 2013. 
[35] S. D. Galbraith, Equations for Modular Curves, DPhil thesis, University of Oxford, 1996.

[36] T. Gee, Automorphic lifts of prescribed types, Mathematische Annalen 350 (2011), 107-144.

[37] E. Halberstadt and A. Kraus, Sur la courbe modulaire $X_{E}(7)$, Experiment. Math. 12 (2003), no. $1,27-40$.

[38] J. Harris and J. H. Silverman, Bielliptic curves and symmetric products, Proceedings of the American Mathematical Society 112 (1991), no. 2, 347-356.

[39] R. Hartshorne, Algebraic Geometry, GTM 52, Springer Verlag, 1977.

[40] F. Jarvis and J. Manoharmayum, On the modularity of supersingular elliptic curves over certain totally real number fields, Journal of Number Theory 128 (2008), no. 3, 589-618.

[41] F. Jarvis and P. Meekin, The Fermat equation over $\mathbb{Q}(\sqrt{2})$, Journal of Number Theory 109 (2004), no. 1, 182-196.

[42] S. Kamienny, Torsion points on elliptic curves and q-coefficients of modular forms, Invent. Math. 109 (1992), no. 2, 221-229.

[43] C. Khare and J.-P. Wintenberger, Serre's modularity conjecture. I, Invent. Math. 178 (2009), no. 3, 485-504.

[44] C. Khare and J.-P. Wintenberger, Serre's modularity conjecture. II, Invent. Math. 178 (2009), no. $3,505-586$.

[45] H. Kisilevsky and M. R. Murty, Elliptic Curves and Related Topics, CRM Proceedings and Lecture Notes 4, American Mathematical Society, 1994.

[46] M. Kisin, Moduli of finite flat group schemes, and modularity, Annals of Mathematics. Second Series 170 (2009), no. 3, 1085-1180.

[47] W. Kuyk and J.-P. Serre (editors), Modular Functions of One Variable III, Lecture Notes in Mathematics 350, Springer-Verlag, 1973.

[48] R. Langlands, Base Change for GL(2), Ann. of Math. Studies 96, Princeton University Press, 1980 .

[49] B. Le Hung, Modularity of elliptic curves over some totally real fields, arXiv:1309.4134, 16 September 2013.

[50] G. Ligozat, Courbes modulaires de niveau 11, pages 147-237 of 67.

[51] J. Manoharmayum, On the modularity of certain $\mathrm{GL}_{2}\left(\mathbb{F}_{7}\right)$ Galois representations, Math. Res. Lett. 8 (2001), no. 5-6, 703-712.

[52] J. Manoharmayum, Serre's conjecture for mod 7 Galois representations pages 141-149 of 21 .

[53] B. Mazur, Rational Points on Modular Curves, pages 107-147 of 67.

[54] B. Mazur, Modular curves and the Eisenstein ideal, Inst. Hautes Études Sci. Publ. Math. 47 (1977), 33-186 (1978).

[55] L. Merel, Bornes pour la torsion des courbes elliptiques sur les corps de nombres, Invent. Math. 124 (1996), no. 1-3, 437-449.

[56] J. S. Milne, Abelian Varieties, Chapter V of [18.

[57] G. Muić, On embeddings of modular curves in projective space, preprint, 17 March 2012.

[58] B. Poonen, E. F. Schaefer and M. Stoll, Twists of X(7) and primitive solutions to $x^{2}+y^{3}=z^{7}$, Duke Math. J. 137 (2007), 103-158.

[59] A. Ogg, Hyperelliptic modular curves, Bulletin de la Société Mathématique de France 102 (1974), 449-462.

[60] K. A. Ribet, Abelian varieties over $\mathbb{Q}$ and modular forms pages 241-261 of [21].

[61] D. E. Rohrlich, Elliptic curves and the Weil-Deligne group, pages 125-158 of 45.

[62] D. E. Rohrlich, Modular curves, Hecke correspondences, and L-functions, pages 41-100 of [19.

[63] K. Rubin, Modularity of mod 5 representations, pages 463-474.

[64] J.-P. Serre, Properiétés galoisiennes des points d'ordre fini des courbes elliptiques, Inventiones Math. 15 (1972), 259-331.

[65] J.-P. Serre, Lectures on the Mordell-Weil Theorem, Aspects of Mathematics 15, Vieweg, 3rd edition, 1997.

[66] J.-P. Serre, Topics in Galois Theory, A K Peters/CRC Press, 2nd edition, 2007.

[67] J.-P. Serre and D. B. Zagier (editors), Modular Functions of One Variable V, Lecture Notes in Mathematics 601, Springer-Verlag, 1977.

[68] S. Siksek, The modular approach to Diophantine equations, pages 495-527 of [16].

[69] S. Siksek, Chabauty for symmetric powers of curves, Algebra \& Number Theory 3 (2009), no. 2, 209-236. 
[70] S. Siksek, Explicit Chabauty over number fields, Algebra \& Number Theory 7 (2013), no. 4, $765-793$.

[71] J. H. Silverman, Advanced Topics in the Arithmetic of Elliptic Curves, GTM 151, Springer, 1994.

[72] C. Skinner and A. Wiles Residually reducible representations and modular forms, Publications mathématiques de l'I.H.É.S. 89 (1999), 5-126.

[73] C. Skinner and A. Wiles Nearly ordinary deformations of irreducible residual representations, Ann. Fac. Sci. Toulouse Math. 10, (2001), 185-215.

[74] W. A. Stein, Modular Forms: A Computational Approach, American Mathematical Society, 2007.

[75] M. Stoll, Implementing 2-descent for Jacobians of hyperelliptic curves, Acta Arith. 98 (2001), $245-277$.

[76] M. Stoll, Rational points on curves, Journal de Théorie des Nombres de Bordeaux 23 (2011), $257-277$.

[77] A. V. Sutherland, A local-global principle for rational isogenies of prime degree, Journal de théorie des nombres de Bordeaux 24 (2012), no. 2, 475-485.

[78] H. P. F. Swinnerton-Dyer, On $\ell$-adic representations and congruences for coefficients of modular forms, pages $1-55$ of 47 .

[79] R. Taylor, On Galois representations associated to Hilbert modular forms, Inventiones Mathematicae 98 (1989), no. 2, 265-280.

[80] R. Taylor On icosahedral Artin representations II, American Journal of Mathematics 125 (2003), 549-566.

[81] R. Taylor and A. Wiles, Ring-theoretic properties of certain Hecke algebras, Annals of Mathematics 141 (1995), no. 3, 553-572.

[82] J. Tunnell, Artin's conjecture for representations of octahedral type, Bull. A.M.S. 5 (1981), $173-175$.

[83] A. Wiles, Modular elliptic curves and Fermat's Last Theorem, Annals of Mathematics 141 (1995), no. 3, 443-551.

[84] A. Wiles, On ordinary $\lambda$-adic representations associated to modular forms, Invent. Math. 94 (1988), no. 3, 529-573.

Mathematisches Institut, Universität Bayreuth, 95440 Bayreuth, Germany

E-mail address: nunobfreitas@gmail.com

Department of Mathematics, Harvard University, One Oxford Street, Cambridge, MA 02138, USA

E-mail address: lhvietbao@googlemail.com

Mathematics Institute, University of Warwick, CV4 7AL, United Kingdom

E-mail address: samir.siksek@gmail.com 